
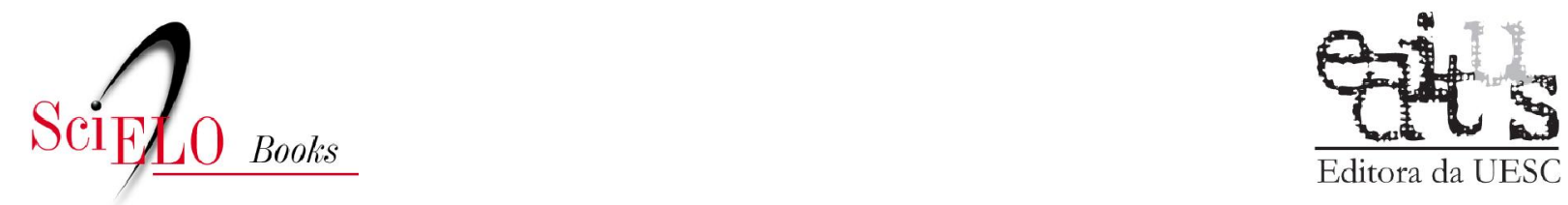

Editora da UESC

\title{
27 - Parasitoides e outros inimigos naturais das formigas Poneromorfas
}

\author{
Thalles Platiny Lavinscky Pereira \\ Juliana Martins da Silva-Freitas \\ Freddy Ruben Bravo
}

\section{SciELO Books / SciELO Livros / SciELO Libros}

PEREIRA, TPL., SILVA-FREITAS, JM., and BRAVO, FR. Parasitoides e outros inimigos naturais das formigas Poneromorfas. In: DELABIE, JHC., et al., orgs. As formigas poneromorfas do Brasil [online]. Ilhéus, BA: Editus, 2015, pp. 403-424. ISBN 978-85-7455-441-9. Available from SciELO Books $<\underline{\text { http://books.scielo.org }>\text {. }}$

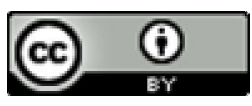

All the contents of this work, except where otherwise noted, is licensed under a Creative Commons Attribution 4.0 International license.

Todo o conteúdo deste trabalho, exceto quando houver ressalva, é publicado sob a licença Creative Commons Atribição 4.0.

Todo el contenido de esta obra, excepto donde se indique lo contrario, está bajo licencia de la licencia Creative Commons Reconocimento 4.0. 


\title{
Parasitoides e outros inimigos naturais das formigas Poneromorfas
}

\author{
Thalles Platiny Lavinscky Pereira, \\ Juliana Martins da Silva-Freitas, Freddy Ruben Bravo
}

\section{Resumo}

Alguns grupos de seres vivos são capazes de burlar as defesas das formigas e de suas colônias, invadindo e permanecendo nos ninhos, algumas vezes estabelecendo relações de parasitismo e/ou parasitoidismo com as mesmas. Entre os organismos classificados como endo/ectoparasitas das formigas estão: vírus, bactérias, fungos, protozoários, nematódeos, platelmintos, ácaros e insetos. Em relação às poneromorfas, até o momento, são conhecidas interações parasíticas com fungos, nematódeos, platelmintos, ácaros e insetos. Neste capítulo trataremos de todos estes grupos (exceto ácaros), mas daremos ênfase especial aos insetos das ordens Diptera e Hymenoptera. Dentro da ordem Diptera, a família Phoridae é a mais representativa no que se refere às interações de parasitoidismo com formigas poneromorfas. Esta família distribuise por todas as regiões biogeográficas e reúne 4 mil espécies em 289 gêneros. Somente os gêneros Megaselia e Apocephalus foram, até o momento, confirmados como parasitoides diretos de formigas poneromorfas dos gêneros Dinoponera, Ectatomma, Gnamptogenys, Leptogenys, Neoponera, Odontomachus, Pachycondyla e Paraponera. Ainda entre os dípteros, espécies do gênero Bengalia, da família Calliphoridae, foram registrados praticando cleptoparasitismo de operárias do gênero Bothroponera que estavam forrageando. Em relação aos himenópteros, membros de duas superfamílias foram, até o momento, relatados como inimigos naturais que estabeleceram algum tipo de relação parasítica com as formigas poneromorfas: Chalcidoidea e Vespoidea. De acordo com os registros realizados até o ano de 2012, a superfamília Chalcidoidea inclui mais de $70 \%$ de todas as espécies de vespas que parasitam formigas, e para as formigas poneromorfas, até o momento, foram registradas três famílias de vespas parasitoides que as atacam: Encyrtidae, Eucharitidae e Perilampidae. No Brasil, somente um estudo registrando essas interações foi realizado até agora. O parasitismo social também tem sido registrado entre as formigas poneromorfas, sendo esse comportamento relatado em pelo menos cinco subfamílias de Formicidae: Dolichoderinae, Myrmicinae, Formicinae, Pseudomyrmecinae e Ectatomminae. Esses taxons foram observados se desenvolvendo à custa de poneromorfas dos gêneros Rhytidoponera, Ectatomma e Diacamma. Os diversos estudos que tratam das relações parasíticas entre poneromorfas e outros organismos mostram que através de uma série de comportamentos complexos e adaptações

PEREIRA, Thalles Platiny Lavinscky; SILVA-FREITAS, Juliana Martins da; BRAVO, Freddy Ruben. Parasitoides e outros inimigos naturais das formigas Poneromorfas. In: DELABIE, Jacques H. C. et al. As formigas poneromorfas do Brasil. Ilhéus: Editus, 2015. p. 403-424. 
morfológicas e/ou químicas, os inimigos naturais das formigas conseguem contornar os mecanismos de defesa das colônias e atacar as larvas, pupas e adultos. Os comportamentos destes ecto/ endoparasitas podem ser classificados de diversas maneiras, mas os mesmos não se comportam sempre das mesmas formas e as interações ecológicas podem variar no tempo e espaço, de forma que classificá-los de maneira rigorosa torna-se uma tarefa complexa. Além disso, tais comportamentos não foram completamente elucidados e percebe-se a necessidade de estudos no que tange à biologia das interações entre os organismos e as formigas poneromorfas. Uma vez que o conhecimento sobre as interações entre os mais diversos grupos aumenta e reúne grande parte do conhecimento disponível acerca do assunto, é possível levantar questões sobre a coevolução desses organismos, aspectos de sua biologia e comportamento e, em alguns casos, até sobre sua utilização no controle biológico.

\section{Abstract}

Parasites and other natural enemies of poneromorph ants - Several animal groups are able to circumvent the extensive defenses of ants and their colonies, invading and residing within the nests, and sometimes establishing parasitic relationships with them. Among the organisms classified as parasites of ants are members of the: viruses, bacteria, fungi, protozoa, nematodes, platyhelminthes, mites and insects. To date, parasitic interactions with poneromorph ants are known with fungi, nematodes, platyhelminthes, mites and insects. This chapter deals with all these groups (except mites), but mainly considers the Diptera and Hymenoptera insect orders. In the Diptera, the Phoridae family is the most representative in respect of the parasitic interaction with poneromorph ants. This family is spread over all biogeographic regions and contains over four thousand species and 289 genera. Only the genera Megaselia and Apocephalus have been confirmed as direct parasitoids of poneromorph ants, they being associated with the ant genera: Dinoponera, Ectatomma, Gnamptogenys, Leptogenys, Neoponera, Odontomachus, Pachycondyla and Paraponera. Also among the Diptera, the genus Bengalia (Calliphoridae) has been recorded exhibiting cleptoparasitic behavior, stealing prey from the workers of the genus Bothroponera that were foraging outside the colony. In relation to the Hymenoptera, members of two superfamilies have been reported as natural enemies that establish types of parasitic relationships with the poneromorph ants: Chalcidoidea and Vespoidea. According to records made up to 2012, the Chalcidoidea superfamily includes more than $70 \%$ of all species of wasps that parasitise ants, with three families of parasitoids known to attack poneromorphs: Encyrtidae, Eucharitidae and Perilampidae. In Brazil, only one record of parasitism has been recorded so far. Social parasitism (or parasitism by other ants) has also been recorded between poneromorph and other ants, and this behavior has been reported in five subfamilies: Dolichoderinae, Myrmicinae, Formicinae, Pseudomyrmecinae and the poneromorph group, the Ectatomminae. These ant groups have been observed developing at the expense of poneromorph genera, like Rhytidoponera, Ectatomma and Diacamma. Studies detailing the parasitic relationship between poneromorphs and other organisms show that through a series of complex behaviours and morphological and/or chemical changes, the natural enemies of ants can circumvent the defense mechanisms of the colonies and attack the larvae, pupae and adults. The behaviour of parasites can be classified in several ways, but they do not always behave in the same ways and ecological interactions may vary in time and space, so classifying them accurately can be a complicated task. Furthermore, these behaviours have not been fully clarified, so there is clearly a need for work on the biology of the interactions between organisms and the poneromorph ants. As knowledge of the interactions among the various groups increases, this may raise questions about the co-evolution of these organisms, their biological and behavioural aspects and in some cases their use in biological control. 


\section{Introdução}

As comunidades de formigas (Hymenoptera: Formicidae) são componentes notáveis da fauna terrestre que se fazem presentes nos mais diversos tipos de habitats (WILSON, 1971). Existem espécies de hábitos arborícolas, as que vivem exclusivamente dentro do solo e/ou que expandem seus ninhos para dentro dos troncos apodrecidos; as que residem em grandes troncos de árvores mortas e aquelas que fixam-se em pequenos galhos resultantes da fragmentação de ramos caídos das árvores (PASSERA; ARON, 2005).

Com tal diversificação de habitats, não é de se espantar que esses insetos sociais estabeleçam os mais diferentes tipos de interações ecológicas com diversas classes de artrópodes e em particular com outros insetos.

O nome mirmecofilia é atribuído a diferentes tipos de interações entre formigas e outros animais (KISTNER, 1982). Erich Wasmann (1894) desenvolveu uma classificação pela qual dividiu os mirmecófilos em cinco grupos: "Synechthrans", "Synoeketes", "Symphiles", Trofobiontes e Ecto/endoparasitas (HÖLLDOBLER; WILSON, 1990; PASSERA; ARON, 2005). Esta classificação levou em conta o comportamento e o nível de integração com o sistema social dos hospedeiros e apesar de ter recebido diversas críticas, ainda é usada na literatura (HÖLLDOBLER; WILSON, 1990; PASSERA; ARON, 2005). A seguir segue uma rápida diferenciação entre os cinco tipos de interações mirmecófilas.

"Synechthrans": são, em sua maioria, artrópodes predadores, tratados de forma hostil pelas formigas, mas que vivem dentro das colônias por serem mais ágeis que elas, ou por usarem mecanismos de defesa (como a liberação de substâncias repelentes ou usando suas cutículas espessas como escudo).

"Synoeketes": é composto por tipos de artrópodes que são primariamente necrófagos e predadores; são ignorados por seus hospedeiros porque são ou muito rápidos ou muito lentos e aparentemente possuem odor neutro.

"Symphiles": são organismos referidos como "intimamente e amigavelmente" relacionados com as colônias de formigas, sendo os simbiontes que, de alguma forma, são completamente aceitos por seus hospedeiros, considerados até membros da colônia.

Trofobiontes: é a categoria que inclui hemípteros fitófagos e lagartas da família Lycaenidae (Lepidoptera); esses organismos não são dependentes das formigas para se alimentar, mas produzem exsudatos açucarados (honeydew) e secreções glandulares nutritivas que podem ser consumidos pelas formigas e em troca recebem proteção das mesmas contra parasitas e predadores.

Ecto/endoparasitas: são os parasitas que vivem na superfície do corpo de seus hospedeiros, ou perfuram superficialmente o exoesqueleto e permanecem sugando a hemolinfa; podem ainda penetrar mais profundamente os tecidos e permanecer dentro dos corpos de seus hospedeiros.

Este capítulo trata principalmente dos organismos contidos nesta última classe, isto é, aqueles que são capazes de permanecer nos ninhos, podendo ser parasitas ou parasitoides das formigas. As definições para esses dois comportamentos podem diferir de acordo a literatura consultada, mas de uma maneira geral podemos dizer que os parasitas - cujo comportamento é chamado de parasitismo - estabelecem uma relação obrigatória e permanente com seu hospedeiro, raramente levando-o à morte (SARMIENTO, 2000). Já os parasitoides têm somente o estágio imaturo como parasita de um único hospedeiro, que é usado como fonte de alimento (HÖLLDOBLER; WILSON, 1990; PASSERA; ARON, 2005) e eventualmente é morto pouco antes de chegar ao imago (SARMIENTO, 2000). O comportamento dos parasitoides é comumente chamado de parasitoidismo (MAIA; TAVARES, 2000; NARDI et al., 2006; RODRÍGUEZ; SÁIZ, 2006).

São numerosos os grupos que podem ser classificados como parasitas ou parasitoides de formigas: vírus, bactérias, fungos, protozoários, nematódeos, platelmintos, ácaros e insetos. Em relação às formigas poneromorfas, até o momento, são conhecidas interações parasíticas com fungos, nematódeos, platelmintos, ácaros e insetos. No entanto, esses organismos não se comportam sempre da mesma forma e as interações ecológicas podem variar no tempo e espaço, de forma que classificá-las de maneira rigorosa se torna uma tarefa complicada.

\section{Diversidade e biologia dos Diptera inimigos naturais das formigas poneromorfas}

Diptera é uma das quatro ordens megadiversas de insetos holometábolos, possuindo cerca de 153 mil espécies descritas (THOMPSON, 2008). Na região Neotropical são conhecidas 118 famílias e cerca de 31 mil espécies (AMORIM, 2009). No Brasil já foram descritas aproximadamente 8.700 espécies (CARVALHO et al., 2009). Na ordem 
Diptera, existem relatos de parasitoidismo de Phoridae e cleptoparasitismo de Calliphoridae sobre formigas poneromorfas.

\section{Phoridae}

Phoridae é uma família da ordem Diptera constituída por espécies de tamanho pequeno, entre 0,4 e 6,0 mm de comprimento, geralmente com corpo compacto, frequentemente cerdoso e venação característica, com veias radiais e costal espessas, sem veias transversais (BROWN, 2009). Representantes da família são encontrados em todas as regiões biogeográficas e reúnem 4 mil espécies em 289 gêneros (THOMPSON, 2008), com aproximadamente 850 espécies no Brasil (AMENT; PEREIRA, em preparação).

As espécies de Phoridae apresentam extensa plasticidade quanto a ocupação de nichos ecológicos, destacando-se como polinizadoras, minadoras, decompositoras, associadas a fungos, predadoras na fase larval, produtores de miiases, cavernícolas, parasitoides de invertebrados, incluindo: moluscos, aranhas, anelídeos, miriápodes, diplópodes e insetos (DISNEY, 1994, BROWN, 2009).

Com o intuito de localizar seus hospedeiros, os forídeos podem usar informações visuais e sinais químicos. Esses últimos podem ser produzidos através de recrutamento para defesa da colônia, ou para forrageio (ORR et al., 1997), quando as formigas são feridas em combates com outras formigas, ou sofrem predação parcial, como ocorre na interação de Apocephalus paraponerae Borgmeier com a formiga Paraponera clavata (Fabricius) (BROWN; FEENER, 1991).

Alguns autores sugerem que essa atração através de ferimentos é uma forma exclusiva e específica dos forídeos parasitoides das poneromorfas. Essa suposição se dá em função dos estudos realizados até o momento que não encontraram em outros grupos de formigas - como por exemplo em Atta e Solenopsis (Myrmicinae) e Eciton (Dorylinae) - a mesma forma de atração para parasitismo (BROWN; FEENER, 1991).

Dentre os forídeos que são encontrados associados às colônias das poneromorfas podemos citar os parasitoides propriamente ditos, tais como os gêneros Apocephalus Coquillett (Figura 27-1) e Megaselia Rondani, assim como espécies que vivem associadas às colônias das formigas como Cataclinusa Schmitz, Rhynchomicropteron Annandale, Puliciphora Dahl e Woodiphora Schmitz (Tabela 27-1). Dentro desse último grupo, muitas espécies possuem fêmeas ápteras ou braquípteras que geralmente são carregadas pelo macho durante a cópula. Essas últimas espécies são atraídas pelas colônias das formigas, vivendo na maioria das vezes como inquilinas temporárias.

Forídeos do gênero Rhynchomicropteron vivem associados a colônias de formigas e cupins (BROWN, 1992) e foram reportados em associação com as formigas do gênero Leptogenys Roger.

FIGURA 27-1 - Parasitoide Apocephalus sp. (Phoridae). Espécime coletada em Cachoeiras de Macacu - RJ, Brasil.

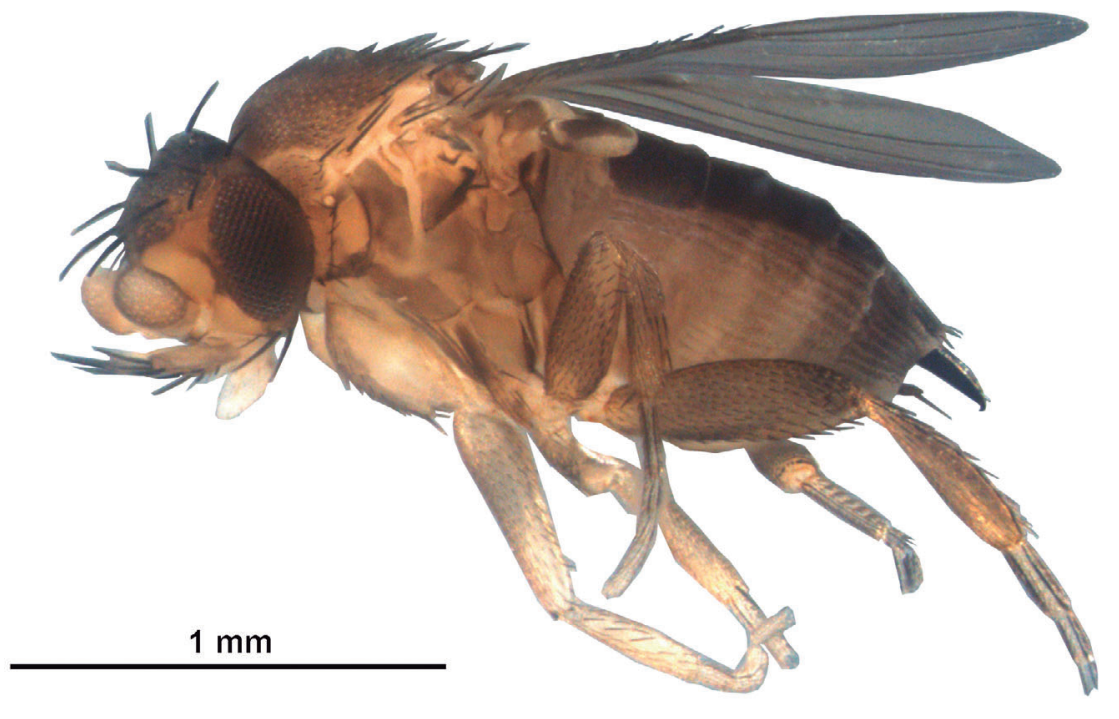


Esses forídeos foram primeiramente classificados por Disney $(1990,1991)$ e Steghaus-Kovac e Disney (1990) como parasitoides dessas formigas, mas, após uma análise minuciosa da morfologia das larvas desse gênero, foi observado que não possuíam as características próprios ao parasitoides e uma função de necrófagos lhes foi atribuída, uma vez que se alimentam dos restos dos detritos produzidos pelas formigas na colônia (BROWN, 1992).

Estudos sobre a morfologia de Megaselia insignicauda Disney, parasitoide da espécie Leptogenys mutabilis (Smith), apontaram que os omatídeos inferiores dos olhos são maiores do que em espécies aparentadas, sugerindo a associação com o hábito de pairar acima do hospedeiro escolhido antes de um ataque de oviposição (DISNEY; SCHROTH, 1989).
Assim como ocorre com frequência nas formigas de correição - Dorylinae - forídeos da espécie Puliciphora rosei Disney foram reportados seguindo a trilha da Ponerinae Leptogenys mutabilis Brown (1992). O gênero Cataclinusa Schmitz foi reportado colocando seus ovos sobre larvas de formigas do gênero Pachycondyla Smith, (WHEELER, 1910). Uma espécie desse mesmo gênero, Cataclinusa pachycondylae (Brues), foi observada em estudos posteriores como um ectoparasita de Pachycondyla harpax (Fabricius), nos quais suas larvas ficavam aderidas às larvas da formiga, roubando o alimento recebido (Figura 27-2) (DISNEY, 1994).

No gênero Dinoponera Roger, Disney descreveu três novas espécies de forídeos associados à

TABELA 27-1 - Forídeos (Diptera-Phoridae) associados às formigas poneromorfas

\begin{tabular}{|c|c|c|c|c|}
\hline \multicolumn{2}{|r|}{ Formiga hospedeira } & \multicolumn{2}{|r|}{ Forídeo (Diptera-Phoridae) } & \multirow[t]{2}{*}{ Referência } \\
\hline Subfamília & Espécies & $\underline{\text { Subfamília }}$ & Espécies & \\
\hline \multirow[t]{5}{*}{ Ectatomminae } & Ectatomma ruidum Roger & \multirow[t]{5}{*}{ Metopininae } & Apocephalus glabriventris Brown & Brown, 2015 \\
\hline & Ectatomma tuberculatum (Olivier) & & Apocephalus lobicauda Brown & Brown, 2015 \\
\hline & Ectatomma tuberculatum (Olivier) & & Apocephalus paraponerae Borgmeier & Brown, 2015 \\
\hline & Gnamptogenys bispinosa (Emery) & & Apocephalus asyndetus Brown & Brown, 2015 \\
\hline & Gnamptogenys sp. & & Apocephalus atrimarginatus Brown & Brown, 2015 \\
\hline \multirow[t]{29}{*}{ Ponerinae } & & \multirow[t]{29}{*}{ Metopininae } & & \\
\hline & Dinoponera gigantea (Perty) & & Apocephalus gigantivorus Brown & Brown, 2015 \\
\hline & Dinoponera gigantea (Perty) & & Apocephalus miricauda Borgmeier & Brown, 2015 \\
\hline & Dinoponera longipes Emery & & Apocephalus dinoponerae Brown & Brown, 2015 \\
\hline & Dinoponera longipes Emery & & Apocephalus kungae Brown & Brown, 2015 \\
\hline & Dinoponera lucida Emery & & Apocephalus exlucida Disney & Disney et al., 2015 \\
\hline & Dinoponera lucida Emery & & Megaselia species A Disney & Disney et al., 2015 \\
\hline & Dinoponera lucida Emery & & Megaselia species B Disney & Disney et al., 2015 \\
\hline & $\begin{array}{l}\text { Leptogenys processionalis distinguenda } \\
\text { (Emery) }\end{array}$ & & Puliciphora rosei Disney & Schmid-Hempel, 1998 \\
\hline & Leptogenys ebenina Forel & & $\begin{array}{l}\text { Puliciphora microphthalma (Schmitz; } \\
\text { Miøberg) }\end{array}$ & Schmid-Hempel, 1998 \\
\hline & Leptogenys mutabilis (Smith) & & Puliciphora rosei Disney & Schmid-Hempel, 1998 \\
\hline & Leptogenys diminuta (Smith) & & Puliciphora knighti (Disney) & Schmid-Hempel, 1998 \\
\hline & Leptogenys mutabilis (Smith) & & Megaselia insignicauda Disney & Schmid-Hempel, 1998 \\
\hline & Leptogenys sp. & & Puliciphora longipes Schmitz por Mjoberg & Schmid-Hempel, 1998 \\
\hline & Leptogenys sp. & & Puliciphora rosei Disney & Schmid-Hempel, 1998 \\
\hline & Neoponera villosa (Fabricius) & & Apocephalus constrictus Brown & Brown, 2015 \\
\hline & Neoponera villosa (Fabricius) & & Apocephalus crassilatus Brown & Brown, 2015 \\
\hline & Neoponera villosa (Fabricius) & & Apocephalus globosus Brown & Brown, 2015 \\
\hline & Neoponera apicalis (Latreille) & & Apocephalus constrictus Brown & Brown, 2015 \\
\hline & Neoponera apicalis (Latreille) & & Apocephalus crassilatus Brown & Brown, 2015 \\
\hline & Neoponera commutata (Roger) & & Apocephalus deceptus Brown & Brown, 2015 \\
\hline & Neoponera crenata (Roger) & & Cataclinusa bucki Schmitz & Schmid-Hempel, 1998 \\
\hline & Neoponera laevigata (Smith) & & Ecitomyia juxtaposita Borgmeier & Schmid-Hempel, 1998 \\
\hline & Neoponera unidentata (Mayr) & & Apocephalus atrimarginatus Brown & Brown, 2015 \\
\hline & Neoponera unidentata (Mayr) & & Apocephalus constrictus Brown & Brown, 2015 \\
\hline & Neoponera unidentata (Mayr) & & Apocephalus crassilatus Brown & Brown, 2015 \\
\hline & Odontomachus affinis Guérin-Méneville & & Lenkoa aurita Borgmeier & Schmid-Hempel, 1998 \\
\hline & Odontomachus bauri Emery & & Apocephalus atrimarginatus Brown & Brown, 2015 \\
\hline & Odontomachus bauri Emery & & Apocephalus atrimarginatus Brown & Brown, 2015 \\
\hline
\end{tabular}




\begin{tabular}{|c|c|c|c|c|}
\hline \multicolumn{2}{|r|}{ Formiga hospedeira } & \multicolumn{2}{|r|}{ Forídeo (Diptera-Phoridae) } & \multirow[t]{2}{*}{ Referência } \\
\hline$\underline{\text { Subfamília }}$ & Espécies & $\underline{\text { Subfamília }}$ & Espécies & \\
\hline \multirow[t]{23}{*}{ Ponerinae } & & \multirow[t]{23}{*}{ Metopininae } & & \\
\hline & Odontomachus chelifer (Latreille) & & Apocephalus atrimarginatus Brown & Brown, 2015 \\
\hline & Odontomachus chelifer (Latreille) & & Apocephalus paldiae Brown & Brown, 2015 \\
\hline & Odontomachus haematodus (L.) & & Apocephalus lopesi Brown & Brown, 2015 \\
\hline & Odontomachus hastatus (Fabr.) & & Apocephalus atrimarginatus Brown & Brown, 2015 \\
\hline & Odontomachus laticeps Roger & & Apocephalus atrimarginatus Brown & Brown, 2015 \\
\hline & Odontomachus simillimus Smith & & Megaselia pagei Disney & Schmid-Hempel, 1998 \\
\hline & Odontomachus simillimus Smith & & Woodiphora pallidinervis Borgmeier & Schmid-Hempel, 1998 \\
\hline & Pachycondyla crassinoda (Latr.) & & Apocephalus atrimarginatus Brown & Brown, 2015 \\
\hline & Pachycondyla crassinoda (Latr.) & & Apocephalus densepilosus Brown & Brown, 2015 \\
\hline & Pachycondyla crassinoda (Latr.) & & Apocephalus trifidus Brown & Brown, 2015 \\
\hline & Pachycondyla harpax (Fabr.) & & Apocephalus atrimarginatus Brown & Brown, 2015 \\
\hline & Pachycondyla harpax (Fabr.) & & Apocephalus eurydomus Brown & Brown, 2015 \\
\hline & Pachycondyla harpax (Fabr.) & & Cataclinusa pachycondylae (Brues) & Schmid-Hempel, 1998 \\
\hline & Pachycondyla impressa (Roger) & & Apocephalus atrimarginatus Brown & Brown, 2015 \\
\hline & Pachycondyla impressa (Roger) & & Apocephalus collatus Brown & Brown, 2015 \\
\hline & Pachycondyla impressa (Roger) & & Apocephalus constrictus Brown & Brown, 2015 \\
\hline & Pachycondyla impressa (Roger) & & Apocephalus contortiventris Brown & Brown, 2015 \\
\hline & Pachycondyla impressa (Roger) & & Apocephalus crassilatus Brown & Brown, 2015 \\
\hline & Pachycondyla impressa (Roger) & & Apocephalus latinsulosus Brown & Brown, 2015 \\
\hline & Pachycondyla striata Smith & & Apocephalus piliventris Borgmeier & Brown, 2015 \\
\hline & Paraponera clavata (Fabricius) & & Apocephalus paraponerae Borgmeier & Schmid-Hempel, 1998 \\
\hline & Paraponera clavata (Fabricius) & & Apocephalus tanyurus Brown & Brown, 2015 \\
\hline \multirow[t]{14}{*}{ Ponerinae } & $\begin{array}{l}\text { Leptogenys processionalis distinguenda } \\
\text { (Emery) }\end{array}$ & \multirow[t]{14}{*}{ Phorinae } & Rhynchomicropteron nudiventer Papp & Schmid-Hempel, 1998 \\
\hline & $\begin{array}{l}\text { Leptogenys processionalis distinguenda } \\
\text { (Emery) }\end{array}$ & & Rhynchomicropteron necaphidiforme Disney & Schmid-Hempel, 1998 \\
\hline & Leptogenys kitteli (Mayr) & & Rhynchomicropteron nudiventer Papp & Schmid-Hempel, 1998 \\
\hline & Leptogenys mutabilis (Smith) & & Rhynchomicropteron nudiventer Papp & Schmid-Hempel, 1998 \\
\hline & Leptogenys processionalis (Jerdon) & & Rhynchomicropteron puliciforme Annandale & Schmid-Hempel, 1998 \\
\hline & Leptogenys cf. mutabilis (Smith) & & $\begin{array}{l}\text { Rhynchomicropteron necaphidiforme } \\
\text { Disney }\end{array}$ & Brown, 1992 \\
\hline & Leptogenys mutabilis (Smith) & & Rhynchomicropteron nudiventer Papp & Brown, 1992 \\
\hline & Leptogenys ocellifera (Roger) & & Rhynchomicropteron puliciforme Annandale & Brown, 1992 \\
\hline & Leptogenys sp. & & Rhynchomicropteron aphidiforme Schmitz & Brown, 1992 \\
\hline & Myopias maligna (Smith) & & Myopiomyia harmani Disney & Schmid-Hempel, 1998 \\
\hline & Odontomachus bauri Emery & & Dohrniphora oricilla Kung; Brown & Brown, 2015 \\
\hline & Odontomachus chelifer (Latreille) & & Dohrniphora longirostrata (Enderlein) & Brown, 2015 \\
\hline & Odontomachus erythrocephalus Emery & & Dohrniphora conlanorum Kung; Brown & Brown, 2015 \\
\hline & Odontomachus rixosus Smith & & Stethopathusa corporaali Schmitz & Schmid-Hempel, 1998 \\
\hline
\end{tabular}

D. lucida Emery (DISNEY et al., 2015). Esse trabalho ratifica uma grande necessidade de pesquisas acerca da biologia das espécies dos hospedeiros e dos inimigos naturais, visto que $D$. lucida se encontra atualmente na lista vermelha de animais em risco de extinção (CAMPIOLO; DELABIE, 2008).

Além dos parasitoides e dos indivíduos que vivem associados às colônias, um comportamento inusitado foi registrado entre o gênero de forídeo Dohrniphora e a Ponerinae do gênero Odontomachus. Nessa interação, os forídeos foram atraídos por odores liberados por feridas provocadas nas formigas e posteriormente eles foram flagrados decapitando-as. Foi verificado que as fêmeas dos forídeos não possuíam ovos fecundados para ovipor na cabeça das formigas, excluindo assim a possibilidade de um processo de parasitismo e classificando-as como saprófagas (BROWN, 2015).

\section{Calliphoridae}

A família Calliphoridae é composta por dípteros caliptrados e têm distribuição mundial. Até o ano de 2008, 1.526 espécies distribuídas em 108 gêneros estavam descritas (THOMPSON, 2008), sendo 126 espécies descritas para a Região Neotropical (AMORIM et al., 2002). Muitas espécies desta família possuem importância médica, veterinária e sanitária (CARVALHO et al., 2009). 
FIGURA 27-2 - Larva de Cataclinusa pachycondylae praticando ectoparasitismo em larva de Pachycondyla harpax. Redesenhado de Disney (1994)

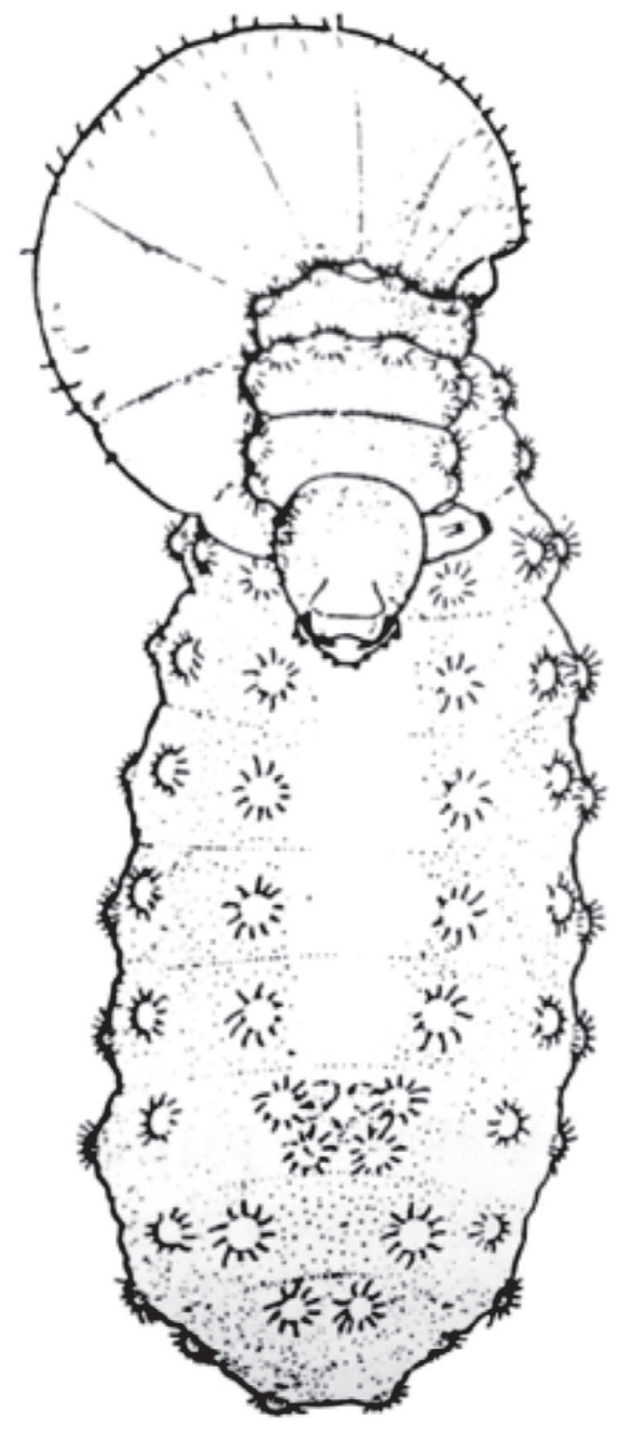

Diferente dessas espécies de interesse médico-forense, e atuando como um inimigo natural, o gênero Bengalia Robineau-Desvoidy já foi registrado praticando cleptoparasitismo, roubando presas de operárias do gênero Bothroponera Mayr que estavam forrageando fora da colônia (MASCHWITZ; SCHONEGGE, 1980). Para esse ataque, as moscas ficam geralmente na vegetação ou em pedras próximas a trilha das formigas para emboscá-las (ROGNES, 2009).

\section{Diversidade e biologia dos Hymenoptera inimigos naturais de formigas poneromorfas}

A ordem Hymenoptera é um dos táxons de insetos mais abundantes e diversificados, com aproximadamente 119 mil espécies descritas ao redor do mundo (HOL, 2014). Na Região Neotropical, segundo Fernández (2002), este número chega a 24 mil espécies, que são distribuídas em 76 famílias. Estas famílias são tradicionalmente distribuídas em duas subordens: Symphyta, que é um agrupamento artificial de himenópteros considerados mais basais; e os Apocrita, que são os himenópteros mais amplamente estudados (como abelhas, vespas e formigas, por exemplo). Este último ainda se subdivide em duas infraordens (ou seções): Parasitica (Terebrantes) e Aculeata (GAULD; BOLTON, 1988; GRIMALDI; ENGEL, 2005). Estudos filogenéticos, no entanto, têm proposto que tanto Symphyta como Parasitica são grupamentos artificiais (parafiléticos), enquanto os Apocrita são amplamente aceitos como um táxon monofilético (NIEVES-ALDREY et al., 2006). Porém, essas denominações ainda são úteis para separar os grandes grupos de himenópteros e ainda são usados por muitos autores (MASON et al. 2006; GOULET; HUBER, 1993).

Em todos esses grupos de himenópteros podem ser encontrados insetos com hábitos altamente diferenciados como os parasitas, os ecto, endo e hiperparasitoides, formadores de galhas (tumores em plantas), herbívoros, polinizadores, predadores, além de espécies eussociais (GODFRAY, 1994; AUSTIN; DOWTON, 2000; DALMOLIN et al., 2004; GRIMALDI; ENGEL, 2005).

De acordo com Mason et al. (2006), cerca de $75 \%$ de todos os apócritos são parasitoides de insetos ou aranhas em seu estágio larval. Quando alcança a maturidade, o parasitoide emerge como um adulto de vida livre, que pode ser carnívoro ou se alimentar de néctar, pólen etc (HÖLLDOBLER; WILSON, 1990; PASSERA; ARON, 2005). Muitos desses parasitoides têm sido relacionados com formicídeos de diversas subfamílias. De acordo com as extensas revisões feitas por Lachaud e Pérez-Lachaud $(2012 ; 2015)$, apesar do variado e sofisticado sistema de defesa das formigas, até o momento já foram detectadas 138 espécies de himenópteros parasitoides que as atacam, distribuídas em três superfamílias: Chalcidoidea, Ichneumonoidea e Diaprioidea.

Existem ainda casos relatados para o parasitismo social entre os himenópteros. Esse padrão comportamental é observado em vários grupos de espécies das famílias Vespidae e Formicidae (BUSCHINGER, 2009), sendo que novos casos de parasitismo social continuam a ser descritos a 
cada ano (BOER; NOORDIJK, 2005, HORA et al., 2005a; ADAMS; LONGINO, 2007), o que sugere que, até o momento, é conhecida apenas uma pequena fração da fauna de parasitas de organismos sociais que existe no Planeta (HÖLLDOBLER; WILSON, 1990).

\section{Parasitoides de formigas}

Entre as subfamílias de formigas que compõem o agrupamento das poneromorfas, as relações de parasitoidismo são pouco conhecidas e até o momento só foram registradas interações diretas de poneromorfas com membros da superfamília Chalcidoidea (Tabela 27-2). No Brasil, os registros dessas interações são ainda mais escassos, com somente uma publicação até o presente momento (BUYS et al., 2010). No entanto, outros registros foram feitos, mas ainda não publicados (SANTOS et al. em preparação).

\section{Superfamília Chalcidoidea}

Chalcidoidea é um grupo megadiverso de insetos, cujas espécies muitas vezes possuem brilho metálico e tamanho reduzido (os menores variam de $1-4 \mathrm{~mm}$ e os maiores podem chegar a $45 \mathrm{~mm}$ ), podendo possuir asas ou não. Quando as asas estão presentes, elas têm uma venação reduzida, sem células completamente fechadas (MUNRO, et al., 2011; CNC, 2013).

Existem cerca de 22 mil espécies descritas de Chalcidoidea, que estão distribuídas em 2 mil gêneros e aproximadamente 20 famílias (NOYES, 2003; CNC, 2013). Apesar dessa grande diversidade numérica, estrutural e biológica (GIBSON, 2006), análises morfológicas e moleculares indicam que a superfamília Chalcidoidea é monofilética (GIBSON, 2006; MUNRO, et al., 2011; HERATY et al., 2012), mas existem problemas nas relações evolutivas entre suas famílias e até mesmo dentro das próprias famílias (MUNRO et al., 2011; HERATY et al., 2012). Assim, o relativamente grande número de famílias (se comparado a Ichneumonoidea, por exemplo, que possui somente duas) pode estar refletindo tanto a maior diversidade morfológica do grupo, como também a falta de entendimento de sua história evolutiva (GIBSON et al., 1999; GIBSON, 2006).

Existem vários calcidoideos que são fitófagos ou predadores, mas a maioria das famílias é parasitoide (CNC, 2013), atacando praticamente todas as ordens e todas as fazes de vida dos insetos (GRISSELL; SCHAUFF, 1997). Inclusive, existem espécies da família Aphelinidae que podem parasitar o sexo oposto de seu próprio táxon (WOOLLEY, 1997).

De acordo com os registros realizados até o ano de 2012, esta superfamília inclui mais do que $70 \%$ de todas as espécies de vespas que parasitam formigas (LACHAUD; PEREZ-LACHAUD, 2012). Em relação às poneromorfas, até o momento foram registradas três famílias de parasitoides que as atacam: Encyrtidae, Eucharitidae e Perilampidae (Tabela 27-2).

\section{Encyrtidae}

Encyrtidae é uma família cosmopolita (NOYES, 2006) e com a maior diversidade estrutural e numérica dos Chalcidoidea: existem cerca de 745 gêneros e 3.825 espécies descritas (NOYES; HANSON, 2006). Destas, estima-se que 565 espécies e aproximadamente 180 gêneros podem ser encontrados na Região Neotropical (NOYES, 2006). No entanto, o grupo é pouco estudado e estes números podem ser bem maiores, visto que extrapolações realizadas a partir da fauna da Costa Rica - que é considerada como uma das mais bem estudadas - sugerem que mais de 15 mil espécies podem ocorrer somente na Região Neotropical (NOYES, 2006).

Os indivíduos adultos dessa família têm entre 0.3 e $0.5 \mathrm{~mm}$, possuindo uma veia marginal muito curta na asa anterior (Figura 27-3) (GIBSON, 1993; TRJAPITZIN, 2008). Seus habitats são os mais variados, mas são particularmente diversos em baixas altitudes, no dossel e em áreas muito secas (NOYES, 2006).

Os imaturos, em sua maioria, sobrevivem parasitando cerca de 150 famílias de artrópodes, mas são principalmente endoparasitoides de diversas ordens de insetos (NOYES, 2006), sendo uma das mais importantes famílias para o controle biológico desses animais - cerca de 40 espécies de Encyrtidae foram introduzidas na Região Neotropical para fins de controle biológico (NOYES; HAYAT, 1994; TRJAPITZIN, 2008; NOYES, 2000; 2012). No entanto, a especificidade em relação ao hospedeiro de muitos Encyrtidae permanece incerta, pois alguns membros da família parecem se desenvolver muito bem em diferentes espécies de insetos, enquanto outras parecem ser parasitas de um hospedeiro específico (NOYES; HAYAT, 
FIGURA 27-3 - Representante do gênero Metaphycus Mercet (Encyrtidae). Espécime coletado em Santa Maria do Jetibá, Espírito Santo, Brasil

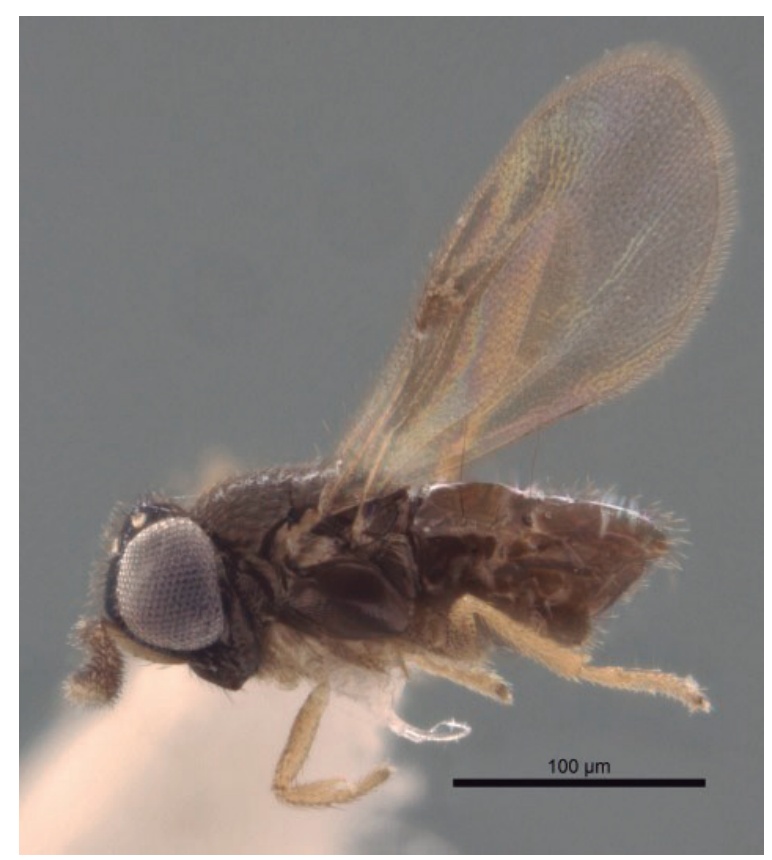

1994; GUERRIERI; NOYES, 2000; NOYES, 2000). Porém, presume-se que muitos grupos parasitam hospedeiros de um grupo restrito - como Hymenoptera ou Lepidoptera, por exemplo (NOYES, 2006). Na maioria das vezes, as espécies são ovipositadas no hospedeiro em seu estágio de larva ou ninfa, mas são conhecidos algumas espécies que ovipositam na pupa ou até mesmo nos adultos (NOYES, 2006).

Em relação aos formicídeos, poucos Encyrtidae têm sido relatados como parasitoides diretos (PEREZ-LACHAUD et al., 2012; 2015), de maneira que existem poucas informações publicadas a respeito da biologia e comportamento dos Encyrtidae parasitoides desses insetos. Os poucos estudos existentes na literatura que analisam interações de Encyrtidae com formigas ou mesmo com outras ordens de insetos indicam que as fêmeas desses parasitoides são cuidadosas na escolha do ninho, do indivíduo e até mesmo da fase de vida do hospedeiro que vão atacar (WHEELER, 1907; STRAND, 1989; ZAPPALÀ et al., 2007).

Somente um registro dessas interações foi realizado com poneromorfas até o momento (Tabela 27-2). Tal registro foi feito por Peréz-Lachaud et al. (2012) na Guiana Francesa, onde observaram pupas da formiga arborícola Neoponera goeldii Forel parasitadas por Blanchardiscus pollux Noyes. De acordo com essas observações, $B$. pollux é um endoparasitoide gregário (apesar da maioria dos parasitoides de Encyrtidae serem solitários). A oviposição ocorre geralmente na parte dorsal e anterior do gáster, próximo ao pecíolo da pupa (PERÉZ-LACHAUD et al., 2012). Ali, eles parecem formar uma pequena massa compacta e quando estão prontos para deixar seu hospedeiro, saem todos por um mesmo orifício pequeno (provavelmente aberto pelas peças bucais de um deles) que é feito na pupa. Outras questões da biologia desses parasitoides - como mecanismos de oviposição e engano das defesas das colônias de formigas - permanecem desconhecidas, visto que esse é o primeiro registro de hospedeiros do gênero Blanchardiscus. De fato, este gênero é pouco conhecido, e até o momento, só duas espécies foram descritas.

\section{Eucharitidae}

A família Eucharitidae é distribuída por quase todas as regiões zoogeográficas (ainda não existe registro na Nova Zelândia e em algumas pequenas ilhas oceânicas) (HERATY, 2002), formando um grupamento monofilético de animais pequenos ( 2 a $5 \mathrm{~mm}$ ). Suas cores variam do metálico ao preto (ou com padrões de amarelo e preto) e possuem uma asa anterior com veia marginal relativamente longa (Figura 27-4) (HERATY, 2006). Esta é uma das menores famílias dentro da 
FIGURA 27-4 - Representantes da família Eucharitidae: a) gênero Orasema Cameron; b) gênero Kapala Cameron. Espécimes coletados em Conceição da Barra, Espírito Santo, Brasil.

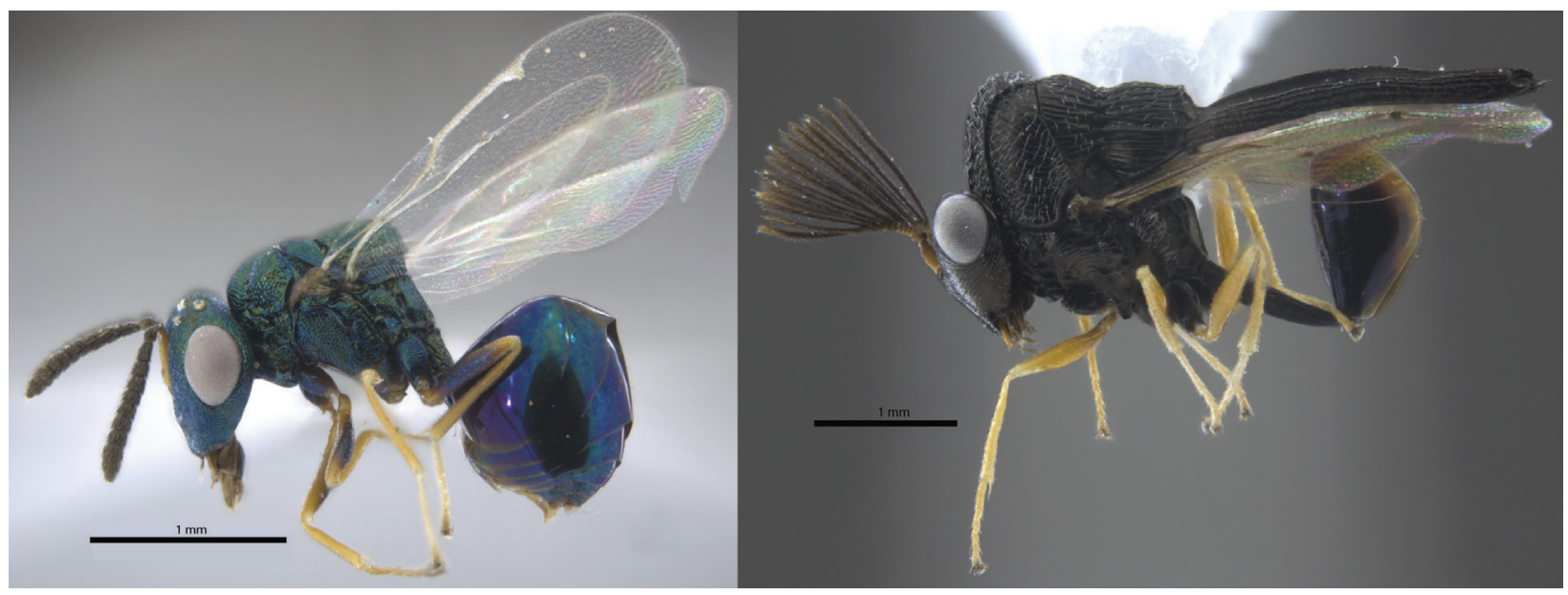

superfamília Chalcidoidea: até o ano de 2014 foram descritos 56 gêneros e menos que 500 espécies (HERATY, 2014). Na Região Neotropical ocorrem duas subfamílias: Oraseminae e Eucharitinae, que englobam aproximadamente 160 espécies e 16 gêneros (HERATY, 2002; HERATY, 2014).

Todos Eucharitidae, cujo hospedeiro é conhecido, parasitam formigas (CLAUSEN, 1940; HERATY, 2002; HERATY et al., 2012; TORRÉNS, 2013). Para as poneromorfas, os trabalhos publicados até o momento mostram que as subfamílias mais atacadas são as Ponerinae e as Ectatomminae (Tabela 27-2).

Os Eucharitidae, ao contrário da maioria das famílias de vespas parasitoides, não depositam seus ovos no ninho do hospedeiro. As fêmeas utilizam seu ovipositor espesso para colocar os ovos (individualmente ou em massa) em incisões feitas nas folhas, flores ou frutos de plantas de diversas famílias, como Asteraceae e Malvaceae (CLAUSEN, 1940; JOHNSON, 1988; TORRÉNS, 2013). Após a eclosão, as larvas chamadas de "planídios" são muito pequenas, geralmente menores do que $0.13 \mathrm{~mm}$, porém muito ativas e com estruturas caudais que possibilitam o salto (HERATY et al., 2004; TORRÉNS, 2013). Para chegar às formigas, que são seu hospedeiro final, essas larvas lançam mão de diferentes comportamentos: tanto podem ser levadas para o ninho se as formigas carregam os frutos em que estão depositadas (HERATY; BARBER, 1990) ou, mais comumente, adotando um comportamento de forésia - utilizando um carregador que pode ser outro parasitoide, algum inseto que sirva de presa para sua formiga hospedeira ou algum outro mirmecófilo (HERATY et al., 2004). Porém, a maioria dos planídios irá utilizar operárias de sua formiga hospedeira que estão forrageando (CLAUSEN, 1940; HERATY et al., 2004; TORRÉNS, 2013), pois é provável que as formigas sejam de alguma maneira atraídas pelas larvas dos parasitoides. A partir de estudos de laboratório, Heraty e Barber (1990) observam que formigas do gênero Camponotus Mayr são atraídas pelo líquido liberado pelas larvas recém-eclodidas, o que resultava na transferência de vários planídios para as partes bucais das formigas, que os carregam até o ninho. Por outro lado, os ovos dos parasitoides são levados por formigas do gênero Paratrechina Motschulsky, a medida que amadurecem, pois há uma secreção apical nesses ovos que poderia ser uma valiosa fonte nutricional para as formigas (HERATY et al., 2004). Uma vez dentro do ninho, o planidio anexa-se a uma larva, mas não se desenvolve até que a mesma chegue ao estágio larval final ou até a fase de pré-pupa (CLAUSEN 1941). Os planídios de Eucharitidae podem ser tanto ectoparasitoides como endoparasitoides da formiga hospedeira: Gollumiellinae e Oraseminae são endoparasitoides, enquanto que as Eucharitinae são ectoparasitoides (WHEELER, 1907; HERATY; MURRAY, 2013).

O tamanho diminuto dos planídios tem sido usado como justificativa para explicar sua entrada e permanência no ninho sem que as formigas o percebam (VANDER MEER et al., 1989). Assim, eles desenvolvem-se como coinobiontes, através de 
três ínstares que se alimentam sobre a região ventral e torácica das pupas hospedeiras (HERATY, 1994; HERATY et al., 2004). Normalmente, apenas um parasitoide se desenvolve por cada pupa, mas pode haver tanto o multiparasitismo como o superparasitismo.

Os parasitoides normalmente emergem das pupas dentro do ninho de seus hospedeiros e podem sair por si só, ou ser carregados para fora das colônias pelas formigas como se fossem lixo (LACHAUD et al., 1998; PÉREZ-LACHAUD et al., 2006a; TORRÉNS, 2013). Se os parasitoides permanecem dentro da colônia por algumas horas, as formigas mostram apenas uma moderada agressividade a eles (LACHAUD; PEREZ-LACHAUD, 2012; LACHAUD et al., 1998), o que pode ser resultado tanto de adaptações morfológicas como de mimetismo químico dos parasitoides (LACHAUD et al., 1998; HOWARD et al., 2001; PÉREZ-LACHAUD et al., 2015). Espécies do gênero Dilochanta, por exemplo, possuem uma depressão no escutelo que pode estar associada a secreções que provavelmente têm alguma relação com a ausência de agressões aos parasitoides em suas primeiras horas de vida dentro do ninho (LACHAUD et al., 1998; HERATY, 1998). Um único caso de Eucharitidae emergindo fora do ninho de seus hospedeiros foi registrado no Brasil por Buys et al. (2010), que observaram pupas parasitadas de Dinoponera lucida Emery sendo carregadas para fora do ninho onde, só então, os parasitoides emergiam.

Uma vez fora da colônia do hospedeiro, os parasitas adultos costumam acasalar logo após a emergência (dentro de algumas horas). Os indivíduos de algumas espécies não se alimentam (JOHNSON, 1988) e vivem poucos dias, de maneira que raramente são capazes de se dispersar por maiores distâncias (CLAUSEN, 1923).

Durante muito tempo acreditou-se que a relação entre Eucharitidae e Formicidae era espécie-específica, mas diversos estudos têm demonstrado que algumas destas vespas podem atacar diferentes gêneros e mesmo diferentes subfamílias de formigas (PÉREZ-LACHAUD, et al., 2006b; LACHAUD et al., 2012). Kapala iridicolor (Cameron), por exemplo, foi observada parasitando espécies de Ectatomma Smith, Gnamptogenys Roger, e Pseudoponera Emery (Tabela 27-2) (PÉREZ-LACHAUD, et al., 2006a; LACHAUD et al., 2012). No entanto, é interessante ressaltar que as espécies de Eucharitidae atacam normalmente gêneros de formigas intimamente relacionados (HERATY, 2002) e existe certa especificidade entre subfamílias de Eucharitidae e Formicidae: Oraseminae são parasitas de Myrmicinae, enquanto Eucharitinae são parasitas de Ponerinae ou Formicinae (HERATY, 1994).

Por último, os efeitos das vespas sobre seus hospedeiros ainda não estão claramente quantificados (MURRAY et al., 2013), embora os adultos e as larvas tenham sido registradas em ninhos de formigas durante todo o ano, com taxas de parasitismo que podem chegar a $25 \%$ do total de pupas (CLAUSEN, 1923; PÉREZ-LACHAUD et al., 2010).

\section{Perilampidae}

São reconhecidos cerca de 280 espécies e 15 gêneros de Perilampidae (UCD, 2004), os quais compreendem três subfamílias: Chrysolampinae, Philomidinae e Perilampinae (HERATY el al., 2012; HERATY; MURRAY, 2013). Apesar de serem morfologicamente distintas, essas são agrupadas na mesma família tendo em vista caracteres morfológicos dos adultos e do primeiro ínstar do estágio larval (DARLING; MILLER 1991). Perilampidae e Eucharitidae formam um grupo monofilético dentro de Chalcidoidea (HERATY et al., 2012; HERATY; MURRAY, 2013).

Os espécimes da família Perilampidae têm entre 1 e $12 \mathrm{~mm}$ de comprimento, com coloração escura que pode ser de metálica a preta, e asas com venação reduzida, cuja veia marginal tem tamanho médio (Figura 27-5).

Diferentemente de Eucharitidae, Perilampidae tem uma maior diversidade de hospedeiros, abrangendo diversas ordens de insetos, como Neuroptera, Coleoptera, Diptera e Hymenoptera (DARLING, 2006). No entanto, interações desses parasitoides com poneromorfas só foram registradas até o momento no sudeste do Peru por Davidson e Fisher (1991). Foi observada a emergência de vários Perilampidae (cerca de nove indivíduos de uma espécie que permanece não identificada) de uma única larva da Ponerinae Neoponera luteola (Roger), que nidifica em troncos de Cecropia membranacea Trécul (Rosales: Urticaceae). De acordo com os autores, os ninhos de $N$. luteola são alvos particularmente fáceis para os parasitoides por que o acesso ao ninho é largo e está aberto para que a rainha da formiga possa recolher os corpúsculos müllerianos. Outros detalhes da biologia e comportamento desses 
FIGURA 27-5 - Representante da família Perilampidae. Espécime coletado em Conceição da Barra, Espírito Santo, Brasil

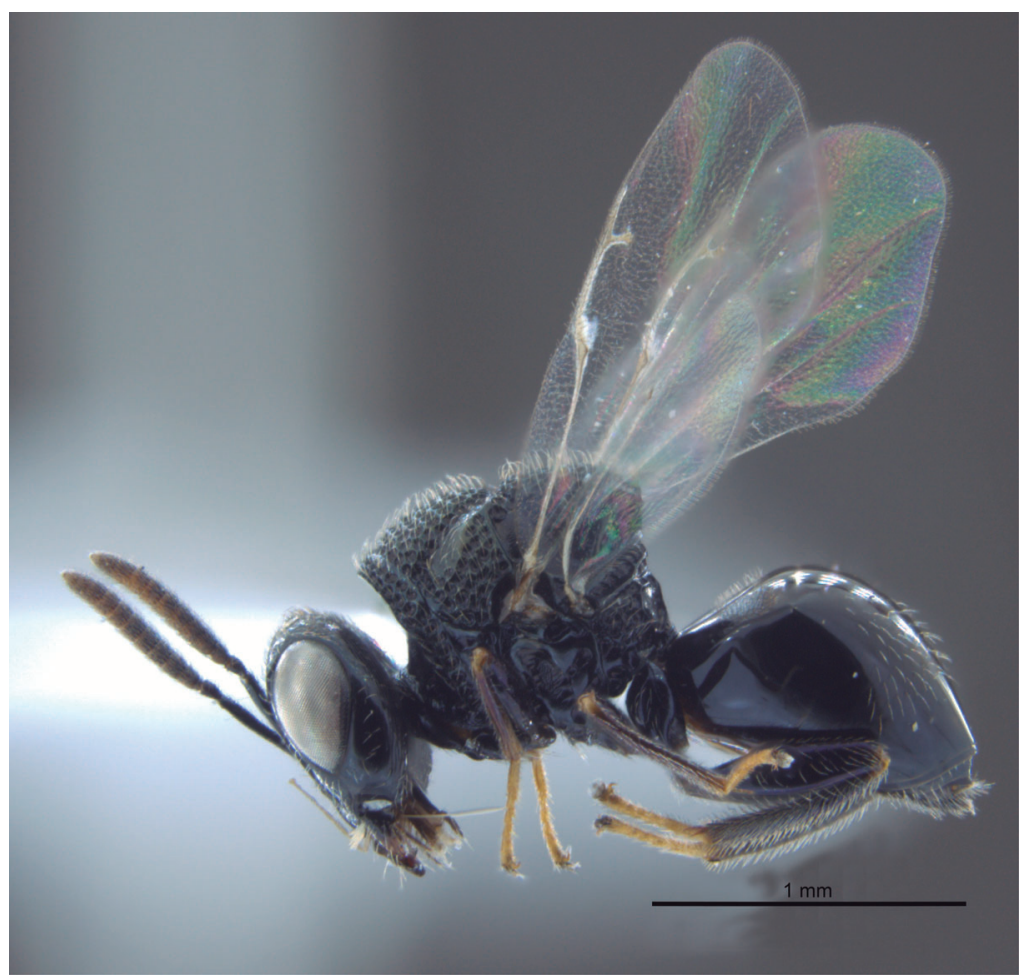

parasitoides não foram detalhados até o momento, mas a literatura descreve essas interações com outros organismos (HERATY; DARLING, 1984; DARLING; MILLER, 1991; DARLING, 1992; HERATY; MURRAY, 2013), observando-se que as características comportamentais e biológicas de Perilampidae assemelham-se àquelas de seu grupo irmão, os Eucharitidae.

Entre as características compartilhadas por essas duas famílias estão a morfologia da larva do tipo planídio e os comportamentos de oviposição das fêmeas (HERATY; DARLING, 1984; DARLING; MILLER, 1991; DARLING, 1992). Uma fêmea adulta coloca cerca de 500 ovos nas imediações do hospedeiro do qual emergiu (HERATY; DARLING, 1984). Ela utiliza seu ovipositor para inserir os ovos dentro do tecido vegetal, principalmente em folhas e frutos, e o primeiro estágio, o planídio, é bastante esclerotizado e ativo, localizando o hospedeiro e aderindo-se a ele. Em muitos casos, o planídio não se alimenta até que o hospedeiro comece sua fase de pupa (DARLING, 2006). A literatura aponta que somente um planídio se desenvolverá em cada larva de formiga (DARLING, 1992; HERATY; MURRAY, 2013), no entanto, Davidson e Fisher (1991) observaram vários Perilampidae emergindo de um único hospedeiro.
O primeiro e o segundo ínstares da larva se desenvolvem como ectoparasitas típicos na região torácica ventral posterior da pupa hospedeira (CLAUSEN 1940; DARLING; MILLER, 1991; DARLING, 1999). As fases posteriores da larva, no entanto, podem ser secundariamente endoparasitas ou hiperparasitas (DARLING; ROBERTS, 1999; HERATY; MURRAY, 2013). Várias espécies da subfamília Perilampinae são hiperparasitoides, desenvolvendo-se em Tachinidae (Diptera) e Ichneumonidae (Hymenoptera) (CLAUSEN, 1940). Algumas espécies podem ser hiperparasitoides obrigatórios, enquanto outras são facultativos (LAING; HERATY, 1981; HERATY; DARLING, 1984).

Todos os Perilampidae completam o seu desenvolvimento após quatro ínstares e são conobiontes (DARLING; MILLER, 1991). Quando se tornam adultos, eles podem ser observados alimentando-se em flores (principalmente espécies da subfamília Perilampinae), mas alguns também se alimentam de "honeydew" (UCD, 2004). Uma fêmea da espécie Perilampus aeneus (Rossius) foi observada perfurando a epiderme de uma folha para se alimentar de exsudato (HERATY; DARLING, 1984). 
TABELA 27-2 - Himenópteros parasitoides registrados em parasitoidismo direto com formigas poneromorfas

\begin{tabular}{|c|c|c|c|c|}
\hline \multicolumn{2}{|r|}{ Formiga hospedeira } & \multicolumn{2}{|r|}{ Himenoptero parasitoide } & \multirow[t]{2}{*}{ Referência } \\
\hline Subfamília & Espécies & Família & Espécie & \\
\hline Ponerinae & Neoponera goeldii (Forel) & \multicolumn{2}{|r|}{ Blanchardiscus sp.? pollux Noyes } & Pérez-Lachaud et al., 2012 \\
\hline \multirow[t]{23}{*}{ Ectatomminae } & Ectatomma brunneum F. Smith & \multirow[t]{51}{*}{ Eucharitidae } & Dicoelothorax platycerus Ashmead & Torréns; Heraty, 2012 \\
\hline & Ectatomma brunneum F. Smith & & Galearia latreillei (Guérin-Méneville) & Torréns, 2013 \\
\hline & Ectatomma brunneum F. Smith & & Kapala sp. & Lachaud et al., 2012 \\
\hline & Ectatomma ruidum Roger & & Kapala iridicolor (Cameron) & $\begin{array}{l}\text { Howard et al., 2001; Pérez-Lachaud et } \\
\text { al.,2006a }\end{array}$ \\
\hline & Ectatomma ruidum Roger & & Kapala izapa Carmichael & Pérez-Lachaud et al., 2006a \\
\hline & Ectatomma tuberculatum (Olivier) & & Dilocantha lachaudii Heraty & Lachaud et al., 1998; Heraty, 1998 \\
\hline & Ectatomma tuberculatum (Olivier) & & Isomerala coronata (Westwood) & Cook, 1905 \\
\hline & Ectatomma tuberculatum (Olivier) & & Kapala sp. & Pérez-Lachaud et al., 2006b \\
\hline & Gnamptogenys menadensis (Mayr) & & Pogonocharis browni Heraty & Heraty, 2002 \\
\hline & Gnamptogenys regularis Mayr & & Kapala iridicolor (Cameron) & Pérez-Lachaud et al., 2006a \\
\hline & Gnamptogenys striatula Mayr & & Kapala iridicolor (Cameron) & Pérez-Lachaud et al., 2006a \\
\hline & Gnamptogenys sulcata (F. Smith) & & Kapala iridicolor (Cameron) & Pérez-Lachaud et al., 2006a \\
\hline & Gnamptogenys sulcata (F. Smith) & & Kapala sp. & Lachaud; Perez-Lachaud, 2001 \\
\hline & Gnamptogenys tortuolosa (F. Smith) & & Kapala sp. & Lachaud et al., 2012 \\
\hline & Rhytidoponera chalybaea Emery & & Chalcura sp. nr. Polita (Girault) & Heraty, 2002 \\
\hline & Rhytidoponera metallica (F. Smith) & & Tricoryna minor (Girault) & Heraty, 2002 \\
\hline & Rhytidoponera metallica (F. Smith) & & Chalcura nigricyanea (Girault) & Heraty, 2002 \\
\hline & Rhytidoponera metallica (F. Smith) & & Chalcura polita (Girault) & Heraty, 2002 \\
\hline & Rhytidoponera sp. & & Tricoryna ectatommae Girault & Girault, 1915 \\
\hline & Rhytidoponera sp. & & Tricoryna iello (Walker) & Heraty, 2002 \\
\hline & Rhytidoponera victoriae (André) & & Tricoryna minor (Girault) & Heraty, 2002 \\
\hline & Rhytidoponera violacea (Forel) & & Tricoryna sp. nr. alcicornis (Boucek) & Heraty, 2002 \\
\hline & $\begin{array}{l}\text { Rhytidoponera metallica var. } \\
\text { critulata (Smith, 1858) }\end{array}$ & & Tricoryna chalcoponerae Brues & Brues, 1934 \\
\hline \multirow[t]{28}{*}{ Ponerinae } & Diacamma scalpratum (F. Smith) & & Schizaspidia diacammae Heraty et al. & Peeters et al. 2015 \\
\hline & Dinoponera lucida Emery & & Kapala sp. & Buys, 2010 \\
\hline & Hypoponera nitidula (Emery) & & Kapala sp. & Mora; Philpott, 2010 \\
\hline & Hypoponera sp. & & Neolosbanus gemma (Girault) & Heraty, 1994 \\
\hline & Hypoponera sp. & & Neolosbanus palgravei (Girault) & Heraty, 1994 \\
\hline & Neoponera apicalis (Latr.) & & Kapala sp. & Lachaud; Perez-Lachaud, 2001 \\
\hline & Odontomachus bauri Emery & & Kapala sp. & Heraty, 2002 \\
\hline & Odontomachus brunneus (Patton) & & Kapala sp. & Lachaud; Perez-Lachaud, 2001 \\
\hline & Odontomachus chelifer (Latreille) & & Latina rugosa (Torréns et al.) & Torréns, 2013 \\
\hline & Odontomachus haematodus (L.) & & Chalcura deprivata (Walker) & Gahan, 1940 \\
\hline & Odontomachus haematodus (L.) & & Schizaspidia convergens (Walker) & Gahan, 1940 \\
\hline & Odontomachus hastatus (Fabr.) & & Kapala sp. & Heraty, 2002 \\
\hline & Odontomachus insularis Guérin-Méneville & & Kapala terminalis Ashmead & Clausen, 1941 \\
\hline & Odontomachus insularis Guérin-Méneville & & Kapala sp. & Clausen, 1941 \\
\hline & Odontomachus laticeps Roger & & Kapala sp. & Lachaud; Perez-Lachaud, 2001 \\
\hline & Odontomachus mayi Mann & & Kapala sp. & Lachaud et al., 2012 \\
\hline & Odontomachus meinerti Forel & & Kapala sp. & Mora; Philpott, 2010 \\
\hline & Odontomachus opaciventris Forel & & Kapala sp. & Lachaud; Perez-Lachaud, 2001 \\
\hline & Odontomachus rixosus F. Smith & & Schizaspidia nasua (Walker) & Heraty, 2002 \\
\hline & Odontomachus ruficeps F. Smith & & Chalcura affinis (Bingham) & Dodd, 1906 \\
\hline & Odontomachus sp. & & Chalcuroides versicolor Girault & Girault, 1913; Boucek, 1988 \\
\hline & Odontomachus troglodytes Santschi & & Ancylotropus sp. & Heraty, 2002 \\
\hline & Pachycondyla crassinoda (Latr.) & & Kapala cuprea Cameron & Myers, 1931 \\
\hline & Pachycondyla harpax (Fabr.) & & Kapala atrata (Walker) & Heraty, 2002 \\
\hline & Pachycondyla harpax (Fabr.) & & Kapala sp. & Mora; Philpott, 2010 \\
\hline & Pseudoponera stigma (Fabr.) & & Kapala iridicolor (Cameron) & Pérez-Lachaud et al., 2006a \\
\hline & Pseudoponera stigma (Fabr.) & & Kapala sp. & Mora; Philpott, 2010 \\
\hline & Neoponera verenae (Forel) & & Kapala sp. & Lachaud et al., 2012 \\
\hline \multirow[t]{2}{*}{ Ponerinae } & & \multirow[t]{2}{*}{ Perilampidae } & & \\
\hline & Neoponera luteola (Roger) & & Unidentified sp. & Davidson; Fisher, 1991 \\
\hline
\end{tabular}




\section{Parasitas sociais de formigas}

Apesar da grande capacidade de organização social de todas as espécies de formigas, muitas vezes elas podem comportar-se como parasitas sociais. Este comportamento é descrito como casos onde espécies sociais se desenvolvem à custa de outra espécie social e de vida livre (HÖLLDOBLER; WILSON, 1990; TINAUT; RUANO, 1999; BUSCHINGER, 2009).

O parasitismo social tem sido relatado em pelo menos quatro subfamílias: Dolichoderinae, Myrmicinae, Formicinae e Pseudomyrmecinae (HÖLLDOBLER; WILSON, 1990); e no ano de 2005, Hora et al. observaram esse comportamento em uma quinta subfamília, as Ectatomminae, sendo este o primeiro caso registrado de uma poneromorfa parasita sociai.

Entre essas subfamílias existem várias modalidades conhecidas de parasitismo social, mas como as espécies nunca vão se comportar exatamente da mesma maneira, torna-se difícil sintetizar todas as modalidades conhecidas (HÖLLDOBLER; WILSON, 1990; TINAUT; RUANO, 1999;). No entanto, é possível distinguir dois comportamentos principais entre as formigas: sociedades compostas e sociedades mistas (sensu TINAUT; RUANO, 1999).

Nas sociedades compostas, duas ou mais espécies vivem muito próximas uma da outra, em alguns casos, usando juntas as mesmas galerias de um ninho (WASMANN, 1891 apud HÖLLDOBLER; WILSON, 1990). Este tipo de relação ecológica abrange diferentes tipos de parasitismo, ocasional ou regular, onde as duas espécies podem roubar recursos alimentares (cleptobiose), predar larvas/pupas (lestobiose) e compartilhar ninhos, mas mantendo ninhadas separadas (parabiose) (BUSCHINGER, 2009). Existem alguns estudos que relatam formicídeos que mantêm este tipo de associação com poneromorfas. Polyrhachis loweryi Kohout, por exemplo, foi observada no ninho de Rhytidoponera sp. em Queensland, Austrália (MASCHWITZ et al., 2003). Esta associação não parece ser obrigatória, pois em situação experimental, operárias de $P$. loweryi foram capazes de coletar alimentos e distribuí-los entre indivíduos coespecíficos via trofalaxia (MASCHWITZ et al., 2003). Outros exemplos de trabalhos que relatam comportamentos desse tipo são Richard et al. (2004) e Wheeler (1986), que documentam associações entre Crematogaster spp. e Ectatomma spp.; e Kaufmann et al. (2003), que registram Strumigenys sp. habitando ninhos de Diacamma sp.

As sociedades mistas tratam de associações onde a prole de duas ou mais espécies está junta e as operárias da espécie hospedeiras tomam conta, pelo menos temporariamente, da prole da espécie parasita (WASMANN, 1891 apud HÖLLDOBLER; WILSON, 1990). Compreende os parasitas temporários, os tomadores de escravos e os inquilinos, o que parece ser o caso da espécie Ectatomma parasiticum Feitosa \& Fresnau, 2008 (FEITOSA et al., 2008). Esta espécie foi encontrada em colônias de Ectatomma tuberculatum (Olivier), em Apazapan, estado de Veracruz, México. Inicialmente,as observações de Hora et al. (2001; 2005a; 2005b) sugeriram que certas microginas presentes nas colônias de E. tuberculatum representavam rainhas de uma outra espécie que seria um parasita social. Estudos taxonômicos, comportamentais e genéticos confirmaram essa hipótese (HORA et al., 2005a; FEITOSA et al., 2008). Tais parasitas sociais parecem desestabilizar a colônia, mas não matam a rainha da colônia hospedeira.

Por outro lado, as formigas poneromorfas também podem travar este tipo de sociedade mista com outras subfamílias não proximamente aparentadas. Maschwitz et al. $(2000 ; 2003)$ documentaram o parasitismo de Polyrhachis lama Kohout em colônias de Diacamma sp. Tal observação foi feita em Java (Indonésia) e os autores notaram que eram as operárias da colônia hospedeira que assumiam, pelo menos em parte, o cuidado com a prole do parasita. Os dados levantados neste trabalho não revelam qualquer prejuízo das colônias hospedeiras de Diacamma e embora não tenha sido testada a habilidade de P. lama em sobreviver e cuidar de sua prole por conta própria, os autores concluem que esta espécie é um parasita obrigatório e permanente de outras espécies de formigas (MASCHWITZ et al., 2000).

Ainda não está claro como as espécies parasitas conseguem burlar a segurança das colônias para roubar recursos ou se integrar às colônias hospedeiras, mas algumas hipóteses são levantadas pelos especialistas que têm analisado esta face do comportamento dos formicídeos. No caso das colônias de E. tuberculatum, talvez o fato de as colônias serem relativamente abertas e com um sistema de reconhecimento relativamente fraco favoreça a intrusão de parasitas sociais (HÖLLDOBLER; WILSON 1990; FÉNÉRON et al., 1999; HORA et al., 2005b). O mimetismo químico também pode 
ocorrer: a autolimpeza das parasitas foi um comportamento frequentemente observado por Hora et al. (2005a), favorecendo a mistura de hidrocarbonetos de parasitas e hospedeiros (D'ETTORRE et al., 2002 apud HORA et al., 2005a).

No entanto, foi notado que as parasitas foram atacadas ocasionalmente por operárias, sugerindo que estas são capazes de discriminar as rainhas parasitas e as de E. tuberculatum (HORA et al., 2005a). Esse comportamento também foi observado entre as Ectatomminae Rhytidoponera sp., onde ataques "brandos" às hospedeiras $P$. loweryi demonstram que o mimetismo químico pode não ser tão eficiente nessa espécie (MASCHWITZ et al., 2003). Esta baixa agressividade ou a dificuldade em reconhecer os intrusos no ninho são vistas como os mais importantes pré-requisitos para a eventual formação deste tipo de associação. Se isso é uma questão de mimetismo químico dos parasitas, ou se as espécies hospedeiras em geral são tolerantes aos intrusos, permanecem uma questão aberta (MASCHWITZ et al., 2000; 2003).

\section{Diversidade e biologia dos outros inimigos naturais de formigas poneromorfas}

\section{Fungos}

São conhecidas aproximadamente $99 \mathrm{mil}$ espécies de fungos (KIRK et al., 2008) e entre eles estão os fungos entomopatogênicos que infectam os insetos: são cerca de 700 espécies distribuídas em 90 gêneros (ROBERTS; HUMBER, 1981). Em relação aos insetos sociais, os estudos publicados até o momento relatam cerca de 60 gêneros que os atacam e destes 25 são patógenos de formigas. Para as formigas poneromorfas, os gêneros Beauveria (Bals.) Vuill, Cordyceps L., Hirsutella Pat. e Laboulbenia Mont.; C.P. Robin são conhecidos como seus parasitas (SCHMID-HEMPEL, 1998).

\section{Platyhelminthes}

Os platelmintos mais derivados são os Neodermata. Estes apresentam a faringe bulbosa e sugadora do ancestral turbelário de vida livre, mas também característica que os permitem a vida parasítica, como a capacidade de reposição parcial ou completa da epiderme celular por uma nova camada sincicial não celular, chamada de neoderme.

Dentre os Neodermata, estão os Cestoda, que possuem 3.400 espécies, sendo todos parasitos finais no trato digestivo de vertebrados (RUPERT et al. 2005). As formigas são hospedeiros intermediários desses parasitos, sendo o cisticerco estágio de desenvolvimento encontrado nelas. Infectadas por esses parasitos, as formigas ficam insaciáveis e com isso sofrem modificação morfológica de extensão do gáster, e mudança de cor (geralmente ficando de marrom escuro à tons amarelados). Por fim, ficam mais lentas e visíveis para os hospedeiros finais, que são algumas aves da família Phasianidae, como pavões, faisões (gêneros Pavo L. e Afropavo L.) e perus (gênero Meleagris L.) (SCHMID-HEMPEL, 1998).

São conhecidos sete gêneros e cerca de 20 espécies de Cestoda que parasitam formigas. Raillietina tetragona Molin já foi encontrada parasitando 32 espécies de Formicidae, com cerca de 1-20 cisticercos por formiga. Essa mesma espécie foi reportada parasitando os gêneros Euponera Forel e Brachyponera Emery (SCHMID-HEMPEL, 1998).

\section{Nemata}

O Filo Nemata ou Nematoda compreende seres blastocelomados, sem segmentação, que são geralmente circulares em seção transversal, com corpos delgados. Existem aproximadamente 25 mil espécies descritas, mas estima-se que existam muito mais (RUPERT et al., 2005; BRUSCA; BRUSCA, 2007). Apesar de serem encontrados em diversos nichos, como generalistas de vida livre, existem aqueles que são parasitas, ocorrendo desta última forma em quase todos os grupos de plantas e animais (BRUSCA; BRUSCA, 2007).

Como parasitas de artrópodes estão, entre outros, os nematódeos da família Mermithidae. Os indivíduos dessa família atacam variadas espécies de aranhas, crustáceos e insetos - destes últimos são capazes de parasitar pelo menos 15 ordens diferentes.

O parasitismo dessa família de nematódeos é geralmente fatal ao hospedeiro. Eles entram em seus corpos em forma de larva e se alimentam de hemolinfa em suas cavidades corporais, passando por várias ecdises, crescendo e atingindo tamanhos que normalmente vão de $0,5 \mathrm{~mm}$ a $10 \mathrm{~mm}$ (no entanto, já foram encontrados mermitídeos de 20 a 50 $\mathrm{cm}$ de comprimento em insetos) (NICKLE, 1972).

Ao serem parasitadas pelos nematoides, as formigas mudam seu comportamento, apresentando fototropismo negativo e um permanente estágio de apetência (BEDDING, 1985). Os mermitídios já foram reportados parasitando as formigas poneromorfas das espécies: Ectatomma tuberculatum (Olivier), Odontomachus chelifer (Latr.), Odontomachus haematodus (L.) 
Paraponera clavata (Fab.), Pachycondyla fuscoatra (Roger),Neoponera inversa (F.Smith), Pachycondyla striata (F. Smith), Ectatomma ruidum Roger e Odontomachus hastatus (Fab.) (POINAR et al. 2006). Por seu difícil reconhecimento taxonômico, apenas os parasitas de E. ruidum e O. hastatus foram identificados, respectivamente Meximermis ectatommi Poinar e Agamomermis costaricensis Poinar (POINAR et al. 2006)

\section{Considerações finais}

Através de uma série de comportamentos complexos e adaptações morfológicas e químicas, os inimigos naturais das formigas conseguem contornar os mecanismos de defesa das colônias e atacar as larvas, pupas e adultos. Esses comportamentos e adaptações são muito complexos e até o momento, ainda não foram completamente elucidados. Sendo assim, percebe-se a necessidade de estudos no que tange à biologia das interações entre os organismos e as formigas poneromorfas. Essas associações reúnem e incrementam grande parte do conhecimento acerca de quais são os mecanismos que possibilitam que elas ocorram, podendo levantar questões sobre a evolução conjunta desses seres e seus aspectos biológicos e comportamentais. Em alguns casos, tais conhecimentos podem ser aplicados, por exemplo, em programas de controle biológico, medida muito usada atualmente, ocupando posição de destaque em programas de Manejo Integrado de Pragas (MIP), seja como responsável pela manutenção do nível de equilíbrio de insetos vistos como pragas, seja como importante medida de controle.

\section{Agradecimentos}

Agradecemos à coleção entomológica da Universidade Federal do Espirito Santo por ceder o material para que as fotos de Chalcidoidea pudessem ser feitas. Este trabalho teve suporte do programa PRONEX , projeto SECTI-FAPESB/ CNPq PNX 001/2009.

\section{Referências}

ADAMS, R.M.; LONGINO, J.T.: Nesting biology of the arboreal fungus-growing ant Cyphomyrmex cornutus and behavioral interactions with the social-parasitic ant Megalomyrmex mondabora. Insectes Sociaux, v. 54, 136-143, 2007.
ALONSO, L.E.; AGOSTI, D. Biodiversity Studies, Monitoring, and Ants: An Overview. In: AGOSTI, D. MAJER, J. D., ALONSO, L. E., SCHULTZ, T. R. Ants: Standard Methods for Measuring and Monitoring Biodiversity. Washington, D. C.: Smithsonian Institution Press, 2000. p. 1-8.

AMENT, D. C.; PEREIRA, T. P. L. Phoridae. Catálogo Taxonômico da Fauna do Brasil. Em preparação.

AMORIM, D. S. Neotropical Diptera diversity: richness, pattems, and perspectives, p. 71 -97. In: PAPE T., BICKEL D., MEIER R., Diptera Diversity: status, challenges and tools. Koninklijke Brill NV, Leiden. 2009. p. 459.

AUSTIN, A.D.; DOWTON, M. Hymenoptera: Evolution, biodiversity and biological control. CSIRO Publishing. 2000. p. 468.

BEDDING, R. A. Nematode parasites of Hymenoptera. In: Plant and Insect Nematodes NICKLE, W.R. (Editor). Marcel Dekker, New York. 1984. pp. 755-795.

BOER, P.; NOORDIJK, J: Myrmica schenckioides nov. sp., a new socially parasitic ant species (Hym., Formicidae).

- Entomologische Berichten v. 65, p. 120-123. 2005.

BOUCEK, Z. Australasian Chalcidoidea (Hymenoptera). A Biosystematic Revision of Genera of Fourteen Families, with a Reclassification of Species. CAB International, Wallingford, UK, 1988.

BROTHERS D. J.; FINNAMORE A. T.; FERNÁNDEZ F. Superfamilia Vespoidea. In: FERNÁNDEZ, F.; SHARKEY M. J. (eds). Introducción a los Hymenoptera de la Región Neotropical. Sociedad Colombiana de Entomología y Universidad Nacional de Colombia, Bogotá, 2006, p. 505-514.

BROTHERS, D. J.; FINNAMORE, A. T. Superfamilia Vespoidea. In: GOULET, H; HUBER, J. T.

Hymenoptera of the World: an Identification Guide to Families. Agriculture Canada. 1993. p. 161-177.

BROWN, B. V. Life history, immature stages and undescribed male of Rhynchomicropteron (Diptera: Phoridae). Journal of Natural History, v. 26, n. 2, p. 407-416, 1992.

BROWN, B.V. Manual of Central American Diptera: Volume 1. NRC, Research Press, Ottawa, Ontario, Canada. 714 p. 2009.

BROWN B. V.; FEENER, D. H. JR. Behavior and host location cues of Apocephalus paraponerae (Diptera: Phoridae), a parasitoid of the giant tropical ant, Paraponera clavata (Hymenoptera: Formicidae). Biotropica v. 23, p. 182-87, 1991. 
BROWN B.; KUNG G.; PORRAS W. A new type of ant-decapitation in the Phoridae (Insecta: Diptera). Biodiversity Data Journal 3: e4299. doi: 10.3897/ BDJ.3.e4299. 2015.

BRUES, C. T. Some new eucharidid parasites of Australian Ants. Bulletin of the Brooklyn Entomological Society, v. 29, p. 201-207. 1934.

BRUSCA, R. C.; BRUSCA, G. J. Invertebrados. 2a.ed., Ed. Guanabara Koogan, Rio de Janeiro. 2007. $968 \mathrm{p}$.

BUSCHINGER, A. Social parasitism among ants: a review (Hymenoptera: Formicidae). Myrmecological News. v. 12. p. 219-235. 2009.

BUYS, S. C.; CASSARO, R.; SALOMON, D. Biological observations on Kapala Cameron 1884 (Hymenoptera Eucharitidae) in parasitic association with Dinoponera lucida Emery 1901(Hymenoptera Formicidae) in Brazil. Tropical Zoology, v. 23, no. 1, p. 29-34, 2010.

CAMPIOLO, S.; DELABIE, J. H. C. Dinoponera lucida Emery. In: Machado, A. B. M., Drummond, G. M.; Paglia, A. P. (Eds.) Livro Vermelho da Fauna Brasileira Ameaçada de Extinção. 1st Ed., Brasília, DF: Ministério do Meio Ambiente; Belo Horizonte, MG: Fundação Biodiversitas. p. 388-389. 2008.

CARVALHO, C. J. B.; RAFAEL, J. A.; COURI, M. S.; SILVA, V. C. Diptera. In: RAFAEL JA, MELO GAR, CARVALHO CJB, CASARI SA, CONSTANTINO $\mathrm{R}$ (editores), Insetos do Brasil: Diversidade e Taxonomia. Ribeirão Preto, Holos Editora, 2012. p. 407-422.

CLAUSEN C.P. The biology of Schizaspidia tenuicornis Ashm., a eucharid parasite of Camponotus. Annals of the Entomological Society of America.16, 195 -219. 1923.

CLAUSEN, C. P. The habits of the Eucharidae. Psyche: A Journal of Entomology v. 48, p. 57-69.1941.

CLAUSEN, C. P. The oviposition habits of the Eucharidae (Hymenoptera). Journal of the Washington Academy of Sciences, v. 30, n. 12, p. 504-516. 1940.

CNC - Canadian National Collection of Insects. Arachnids and Nematodes. http://www.canacoll.org, Acesso em 13 de junho de 2014.

COOK O. F. The social organization and breeding habits of the cotton-protecting Kelep of Guatemala. Technical Series, United States Department of Agriculture, n. 10.p. 1-55. 1905.
DALMOLIN A.; MELO G.A.R.; PERIOTO N.W. Novas espécies de Prodecatoma (Hymenoptera, Eurytomidae) associadas a galhas em frutos de duas espécies de Psidium L. (Myrtaceae), com comentários sobre Prodecatoma spermophaga Costa-Lima. 2004. Revista Brasileira de Entomologia, 48: 519-528. 2004.

DARLING D. C. The life history and larval morphology of Aperilampus (Hymenoptera: Chalcidoidea: Philomidinae), with a discussion of the phylogenetic affinities of the Philominiidae. Systematic Entomology, 17: 331-339. doi: 10.1111/ j.1365-3113.1992.tb00554.x. 1992.

DARLING, D. C. Life history and immature stages of Steffanolampus salicetum (Hymenoptera: Chalcidoidea: Perilampidae). Proceedings of the Entomological Society of Ontario, 130: 3-14. 1999.

DARLING, D. C. Familia Perilampidae. In: FERNÁNDEZ, F.; SHARKEY M.J.( eds). Introducción a los Hymenoptera de la Región Neotropical. Sociedad Colombiana de Entomología y Universidad Nacional de Colombia, Bogotá: 2006. p. 37-56.

DARLING D. C.; MILLER T. D. Life history and larval morphology of Chrysolampus (Hymenoptera: Chalcidoidea: Chrysolampinae) in western North America. Canadian Journal of Zoology 69: 21682177. doi: 10.1139/z91-30. 1991.

DARLING D. C.; ROBERTS H. Life history and larval morphology of Monacon (Hymenoptera: Perilampidae), parasitoids of ambrosia beetles (Coleoptera: Platypodidae). Canadian Journal of Zoology. 77: 1768-1782. 1999

DAVIDSON, D. W.; FISHER, B. F. Symbiosis of ants with Cecropia a function of light regime. In Ant-Plant Interactions, Huxley C. R.; Cutler D. F. (Eds.), Oxford University Press, Oxford, UK. 1991. p. 289-309.

DISNEY, R. H. U. Two new species and a revised key to Rhynchomicropteron (Diptera, Phoridae), Entomologica Fennica, 1, 21-24. 1990.

DISNEY, R. H. L. A new species of Rhynchomicropteron (Dipt., Phoridae) from Nepal. Entomologist's Monthly Magazine, 127, 119-121. 1991.

DISNEY, R.H.L. Scuttle flies: the Phoridae. Chapman and Hall, London, 1994. p. 467.

DISNEY, R. H. L.; SCHROTH, M. Observations on Megaselia persecutrix Schmitz (Diptera: Phoridae) and the significance of ommatidial size-differentiation. Entomologist's Monthly Magazine, v. 124, n. 1500, p. 169-174, 1989. 
DISNEY, R. H. L.; BRAGANÇA, M.AL; TEIXEIRA, M. C. New Species of Scuttle Flies (Diptera: Phoridae) Associated with a Ponerine Ant (Hymenoptera: Formicidae) in Brazil. Sociobiology, v. 62, n. 1, p. 124$127,2015$.

FEITOSA, R. M.; HORA, R. R.; DELABIE, J. H. C.; VALENZUELA, J.; FRESNEAU, D. A new social parasite in the ant genus Ectatomma F. Smith (Hymenoptera, Formicidae, Ectatomminae). Zootaxa 1713: 47-52. 2008.

\section{FÉNÉRON R.; NOWBAHARI E.; DUTROU F.}

Reconnaissance intercoloniale et niveau d'agression chez la fourmi ponérine, Ectatomma tuberculum. Actes des Colloques Insectes Sociaux 12:33-36. 1999.

FERNÁNDEZ, F. Filogenia y sistemática de los himenópteros con aguijón en la Región Neotropical (Hymenoptera: Vespomorpha), In: COSTA, C., S. ;A. VANIN; J. M. LOBO; A. MELIC (eds.). Proyecto de Red Iberoamericana de Biogeografía y Entomología Sistemática. Monografías Tercer Milenio, Zaragoza, España. 2002. Vol. 2. p. 101-138.

GAHAN, A. B. A contribution to the knowledge of the Eucharidae (Hymenoptera: Chalcidoidea). Proceedings of the United States National Museum, vol. 88, p. 425-458. 1940.

GAULD, I.; BOLTON, B. The Hymenoptera. British Museum (Natural History), London. Oxford. 1988. p. 332.

GIBSON, G. A. P. In: Goulet, H; Huber, J. T. Hymenoptera of the World: an identification guide to families. Agriculture Canada. p. 570 - 634. 1993.

GIBSON, G. A. P. Superfamilia Chalcidoidea. En: Fernández, F. y M:J. Sharkey (eds). Introducción a los Hymenoptera de la Región Neotropical. Sociedad Colombiana de Entomología y Universidad Nacional de Colombia, Bogotá: 37-56. 2006.

GIBSON, G. A. P.; HERATY J. M. Y.; WOOLLEY, J. B. Phylogenetics and classification of Chalcidoidea and Mymarommatoidea -A review of current concepts (Hymenoptera, Apocrita). Zoologica Scripta, 28:87124. 1999.

GIRAULT, A. A. New genera and species of chalcidoid Hymenoptera in the South Australian Museum. Transactions of the Royal Society of South Australia, vol. 37, p. 67-115. 1913.

GIRAULT, A. A. Australian Hymenoptera Chalcidoidea-X. Memoirs of the Queensland Museum, vol. 4, p. 225-237. 1915.
GODFRAY H. C. J. Parasitoids: Behavioral and Evolutionary Ecology. Princeton University Press, Princeton, New Jersey. 1994. p. 475.

GOULET, H; HUBER, J. T. Hymenoptera of the World: an identification guide to families. Agriculture Canada. 1993. p. 668.

GRIMALDI, D.; ENGEL, M. S. Evolution of the Insects. Cambridge University Press. ISBN 0-52182149-5. 2005. p. 733.

GRISSELL, E. E.; SCHAUFF M. E.. Chapter 3. Chalcidoidea. In GIBSON, G.A.P., HUBER J.T., WOOLLEY J.B. (eds). Annotated Keys to the Genera of Nearctic Chalcidoidea (Hymenoptera). National Research Council of Canada Research Press, Ottawa, Canada. 1997.p. 45-116.

GUERRIERI, E.; NOYES J. S. Revision of European species of genus Metaphycus Mercet (Hymenoptera: Chalcidoidea: Encyrtidae), parasitoids of scale insects. Systematic Entomology 25: 147-222, 2000.

HERATY J. M. The genus Dilocantha (Hymenoptera: Eucharitidae). Proceedings of the Entomological Society of Washington, vol. 100, no. 1, p. 72-87. 1998.

HERATY, J. Familia Eucharitidae. In: HANSON, P.Y; GAULD, I.D. Hymenoptera de la Región Neotropical. Mem. Amer. Entomol. Institute, Gainesville. 2006.

HERATY J. (2012). Catalog of World Eucharitidae. University of California, Riverside. 108 pp. Disponivel em: <http://hymenoptera.ucr.edu/eucharitidae >. Acesso em: 09 de junho de 2014.

HERATY J. M.; MURRAY E. The life history of Pseudometagea schwarzii, with a discussion of the evolution of endoparasitism and koinobiosis in Eucharitidae and Perilampidae (Chalcidoidea). Journal of Hymenoptera Research. 35: 1-15. doi: 10.3897/JHR.35.6025. 2013.

HERATY, J. M.; BURKS, R. A.; CRUAUD, A.; GIBSON, G. A. P.; LILJEBLAD, J.; MUNRO, J.; RASPLUS, J.-Y.; DELVARE, G.; JANŠTA, P.; GUMOVSKY, A.; HUBER, J.; WOOLLEY, J. B.; KROGMANN, L.; HEYDON, S.; POLASZEK, A.; SCHMIDT, S.; DARLING, D. C.; GATES, M.W.; MOTTERN, J.; MURRAY, E.; DAL MOLIN, A.; TRIAPITSYN, S.; BAUR, H.; PINTO, J. D.; VAN NOORT, S.; GEORGE, J.; YODER, M.; A phylogenetic analysis of the megadiverse Chalcidoidea (Hymenoptera). Cladistics, 29: 466-542. doi: 10.1111/cla.12006. 2012.

HERATY, J. M. The genus Dilocantha (Hymenoptera: Eucharitidae). Proceedings of the Entomological Society of Washington. 100: 72-87. 1998. 
HERATY, J. M. A revision of the genera of Eucharitidae (Hymenoptera: Chalcidoidea) of the world. Memoirs of the American Entomological Institute, v.68, p. $1-367.2002$.

HERATY, J. M.; BARBER, K. N. Biology of Obeza floridana (Ashmead) and Pseudochalcura gibbosa (Provancher) (Hymenoptera: Eucharitidae). Proceedings of the Entomological Society of Washington, v. 92, n. 2, p. 248-258. 1990.

HERATY, J.; HAWKS, D.; KOSTECKI, J. S.; CARMICHAEL, A. Phylogeny and behaviour of the Gollumiellinae, a new subfamily of the antparasitic Eucharitidae (Hymenoptera: Chalcidoidea). Systematic Entomology. Volume 29, Issue 4, pages 544-559. 2004.

HERATY, J. M. A revision of the genera of Eucharitidae (Hymenoptera: Chalcidoidea) of the World. Memoirs of the American Entomological Society, 68, 1-359. 2002.

HERATY, J. M. Classification and evolution of the Oraseminae in the Old World, with revisions of two closely related genera of Eucharitinae (Hymenoptera: Eucharitidae). Life Sciences Contributions, Royal Ontario Museum, 157, 1-174 .1994.

HERATY, J. M.; DARLING, D. C. Comparative morphology of the planidial larvae of Eucharitidae and Perilampidae (Hymenoptera: Chalcidoidea). Systematic Entomology 9(3): 309-328. 1984.

HOL - HYMENOPTERA ONLINE http://hol.osu.edu. Acesso em 21 de maio de 2014.

HÖLLDOBLER, B.; E.O. WILSON. The Ants. Cambridge, Belknap/Harvard University Press,. 1990. p. 732 .

HORA, R. R.; FÉNERÓN, R.; VALENZUELA, J.; FAVILA, M. E.; FRESNEAU, D. Queen-size dimorphism in the ant Ectatomma tuberculatum (Hymenoptera: Formicidae: Ponerinae). Sociobiology, 38, 407-420. 2001.

HORA, R. R.; VILELA, E.; FÉNERÓN, R.; PEZON, A.; FRESNEAU, D.; DELABIE J. Facultative polygyny in Ectatomma tuberculatum (Formicidae: Ectatomminae). Insectes Sociaux, 52: 194-200, 2005a.

HORA, R. R.; DOUMS, C.; POTEAUX, C.; FÉNERÓN, R.; VALENZUELA, J.; HEINZE, J.; FRESNEAU, D. Small queens in the ant Ectatomma tuberculatum: a new case of social parasitism. Behavioral Ecology and Sociobiology, 59, 285-292, 2005b.
HOWARD, R. W.; PEREZ-LACHAUD, G.; LACHAUD, J.-P. Cuticular hydrocarbons of Kapala sulcifacies (Hymenoptera:Eucharitidae) and its host, the ponerine ant Ectatomma ruidum (Hymenoptera: Formicidae). Annals of the Entomological Society of America, v. 94, n. 5, p. 707-716, 2001.

JOHNSON, D. W. Eucharitidae (Hymenoptera: Chalcidoidea): Biology and Potential for Biological Control. The Florida Entomologist, v. 71, n. 4, p. 528-537, 1988.

KAUFMANN, E.; MALSCH, A. K. F.; ERLE, M.; MASCHWITZ, U. Compound nesting of Strumigenys sp. (Myrmicinae) and Diacamma sp. (Ponerinae), and other nesting symbioses of myrmicine and ponerine ants in Southeast Asia. Insectes Sociaux, 50(1), 88-97. 2003.

KIRK, P. M.; CANNON, P. F.; DAVID, J. C.; STALPERS, J.A. Ainsworth and Bisby's Dictionary of the Fungi. 10th ed. CABI. Wallingford. 2008.

KISTNER, D. H. The social insects' bestiary. Social Insects, 3, 1-244. 1982.

LACHAUD, J.-P.; PÉREZ-LACHAUD, G.

Ectaheteromorph ants also host highly diverse parasitic communities: a review of parasitoids of the Neotropical genus Ectatomma. Insectes Sociaux. 2015. 1-12.2015. doi: 10.1007/s00040-015-0390-x.

LACHAUD J.-P.; PEREZ-LACHAUD, G. Fourmis ponerines associees aux parasitoides du genre Kapala Cameron (Hymenoptera, Eucharitidae). Actes des Colloques Insectes Sociaux, v. 14, p. 101-105. 2001.

LACHAUD J. P.; CERDAN, P.; PEREZ-LACHAUD

G. Poneromorph ants associated with parasitoid wasps of the genus Kapala Cameron (Hymenoptera: Eucharitidae) in French Guiana, Psyche: A Journal of Entomology, v. 2012, Article ID 393486. 2012.

LACHAUD J.-P.; PÉREZ-LACHAUD G. Diversity of species and behavior of Hymenopteran parasitoids of ants: a review. Psyche: A Journal of Entomology, v. 2012, p. 1-24. (doi: 10. 1155/2012/134746). 2012.

LACHAUD, J.-P.; PEREZ-LACHAUD, G.; HERATY, J. M. "Parasites associated with the ponerine ant Ectatomma tuberculatum (Hymenoptera: Formicidae): First host record for the genus Dilocantha (Hymenoptera: Eucharitidae), The Florida Entomologist, v. 81, .4, p. 570-574, 1998.

LAING, J.E.; HERATY, J.M. The parasite complex of the overwintering population of Epiblema scudderiana (Lepidoptera: Olethreutidae) in southern Ontario. Proceedings of the Entomological Society of Ontario, 112:59-67. 1981. 
MAIA, V. C.; TAVARES, M. T. Cordiamyia globosa Maia (Diptera, Cecidomyiidae), flutuação populacional e parasitoides (Hymenoptera) associados. Revista Brasileira de Zoologia. v. 17, n. 3, p. 589-593. 2000. doi: 10.1590/S010181752000000300004

MASCHWITZ, U.; DOROW, W. H. O.; BUSCHINGER, A.; KALYTTA, G. Social parasitism involving ants of different subfamilies: Polyrhachis lama (Formicinae) an obligatory inquiline of Diacamma sp. (Ponerinae) in Java. Insectes Sociaux, 47:27-35. 2000.

MASCHWITZ U, SCHÖNEGGE P. Fliegen als Beuteund Bruträuber bei Ameisen. Insects Sociaux, 27:1-4. 1980.

MASCHWITZ, U.; GO, C.; DOROW, W. H. O; BUSCHINGER, A; KOHOUT, R. J. Polyrhachis loweryi (Formicidae): A guest ant parasiting Rhytidoponera sp. (Ponerinae) in Queensland, Australia. Insectes Sociaux, 50:69-76. 2003.

MASON, W. R. M.; HUBER J. T.; FERNÁNDEZ. F. El orden Hymenoptera. In: FERNÁNDEZ, F.; SHARKEY $\mathrm{M}: J$ (eds). Introducción a los Hymenoptera de la Región Neotropical. Sociedad Colombiana de Entomología y Universidad Nacional de Colombia, Bogotá. 2006. p. 505-514.

MORA, A. DE LA; PHILPOTT, S. M. Woodnesting ants and their parasites in forests and coffee agroecosystems. Environmental Entomology, v. 39, n. 5, p. 1473-1481. 2010.

MUNRO, J. B.; HERATY, J.; BURKS, R. A.; HAWKS, D.; MOTTERN, J. L.; CRUAUD, A.; RASPLUS, J.-Y.; JANSTA, P. A molecular phylogeny of the Chalcidoidea (Hymenoptera). PLoS One 6, e27023. 2011.

MURRAY, E. A.; CARMICHAEL, A. E.; HERATY, J. M. Ancient host shifts followed by host conservatism in a group of ant parasitoids. Proceedings of the Royal Society B: Biological Sciences, v. 280, n. 1759, p. 20130495, 2013.

MYERS, J. G. Descriptions and records of parasitic Hymenoptera from British Guiana and the West Indies. Bulletin of Entomological Research, v. 22, p. 267-277. 1931

NARDI, C.; GUERRA, T. M.; ORTH, A. I.; TAVARES, M. T. Himenópteros parasitoides associados a pupas de Methona themisto (Lepidoptera, Nymphalidae) em Florianópolis, Santa Catarina, Brasil. Iheringia, Série Zoologia. v.96, n.3, p. 373-375. 2006 doi: 10.1590/ S0073-47212006000300014.
NICKLE, W. R. A contribution to our knowledge of the Mermithidae (Nematoda). Journal of Nematology, v. 4, n. 2, p. 113, 1972.

NIEVES-ALDREY, J. L.; FONTAL-CAZALLA, F.Y FERNÁNDEZ, Filogenia y Evolución de Hymenoptera. In: FERNÁNDEZ, F.; SHARKEY M:J. (eds). Introducción a los Hymenoptera de la Región Neotropical. Sociedad Colombiana de Entomología y Universidad Nacional de Colombia, Bogotá: 2006. p. 37-56.

NOYES J. S.; HAYAT Y. M. Oriental Mealybug Parasitoids of the Anagyrini (Hymenoptera:

Encyrtidae). CAB International, Oxon, UK.1994. p. 554.

NOYES, J. S. Encyrtidae of Costa Rica (Hymenoptera: Chalcidoidea), 1 . The subfamily Tetracneminae, parasitoids of mealybugs (Homoptera: Pseudococcidae). Memoirs of the American Entomological Institute, 62:1-355. 2000.

NOYES, J. S. 2003.Universal Chalcidoidea database. (URL: http://www.nhm.ac.uk/entomology/chalcidoids/ index.html). Acesso em 29 de maio de 2013.

NOYES, J. S. Famili Encyrtidae. In: FERNÁNDEZ, F.; SHARKEY M.J. (eds). Introducción a los Hymenoptera de la Región Neotropical. Sociedad Colombiana de Entomología y Universidad Nacional de Colombia, Bogotá: 2006. p. 727 - 744.

NOYES, J. S.; HANSON, P. E. Familia Encyrtidae. In: HANSON, P.Y.; GAULD, I.D. Hymenoptera de la Región Neotropical. Memoirs of the American Entomological Institute, Gainesville. 2006. p. 341-351.

ORR, M.; SEIKE, S.; GILBERT, L. Foraging ecology and patterns of diversification in dipteran parasitoids of fire ants in south Brazil. Ecological Entomology, 22(3), 305-314. 1997.

PASSERA, L.; ARON, S. Les Fourmis: Comportement, Organisation Sociale et Évolution. NRC Research Press, 2005. p. 480.

PÉREZ-LACHAUD, G.; LÓPEZ-MÉNDEZA, J. A.; BEUGNONB, G.; WINTERTONC, P.; LACHAUD, J. P. High prevalence but relatively low impact of two eucharitid parasitoids attacking the Neotropical ant Ectatomma tuberculatum (Olivier). Biological Control, v. 52, n. 2, p. 131-139, 2010.

PÉREZ-LACHAUD, G.; HERATY, J. M.; CARMICHAEL, A.; LACHAUD, J. P. Biology and behavior of Kapala (Hymenoptera:Eucharitidae) attacking Ectatomma, Gnamptogenys and Pachycondyla (Formicidae: Ectatomminae and Ponerinae) in Chiapas, Mexico. Annals of the Entomological Society of America, v. 99, n. 3, p. 567-576. 2006 a. 
PÉREZ-LACHAUD, G.; LÓPEZ-MÉNDEZ J. A.; LACHAUD, J. P. Eucharitid parasitism of the Neotropical ant Ectatomma tuberculatum: parasitoid co-occurrence, seasonal variation, and multiparasitism. Biotropica, v. 38, n. 4, p. 574-576. 2006 b.

PÉREZ-LACHAUD, G.; NOYES, J.; LACHAUD, J.P.. First record of an encyrtid wasp (Hymenoptera: Chalcidoidea) as a true primary parasitoid of ants (Hymenoptera: Formicidae). Florida Entomologist, p. 1066-1076. 2012.

POINAR, JR, GEORGE et al. Recent and fossil nematode parasites (Nematoda: Mermithidae) of Neotropical ants. Journal of Invertebrate Pathology, v. 91, n. 1, p. 19-26, 2006.

RICHARD, F.-J., DEJEAN, A.; LACHAUD, J.-P. Sugary food robbing in ants: a case of temporal cleptobiosis. Comptes Rendus Biologies 327: 509-517. 2004.

ROBERTS, D. W.; HUMBER, R. A. Entomogenous fungi. Biology of Conidial Fungi, v. 2, p. 201-236, 1981.

RODRÍGUEZ, F. A.; SÁIZ, F. G. Parasitoidismo de Psyllaephagus pilosus Noyes (Hym.: Encyrtidae) sobre el psílido del eucalipto Ctenarytaina eucalypti (Maskell) (Hem.: Psyllidae) en plantaciones de eucaliptos en la V region. Agric. Téc. v.66 n.4. p. 342-351 2006. doi: 10.4067/S036528072006000400002.

ROGNES, Knut. Revision of the Oriental species of the Bengalia peuhi species group (Diptera, Calliphoridae). Zootaxa, vol. 2251.p. 1-76. 2009.

RUPPERT, E. E., FOX, R. S.; BARNES, R. D.

Zoologia dos Invertebrados. $7^{\mathrm{a}}$ ed., Ed. Roca, São Paulo, 2005. p. 1145

SARMIENTO, F. O. Dicionario de Ecologia Paisajes, Conservacion e Desarrolo Sustentable para Latinoamerica. Editorial Abya-Yala. Ecuador, 2001.514 p.

SCHMID-HEMPEL, P. Parasites in Social Insects. Princeton University Press, 1998. p. 392.

STEGHAUS-KOVAC, S.; DISNEY, R. H. L., A further case of a Rhynchomicropteron (Diptera, Phoridae) associated with a ponerine ant (Hymenoptera, Formicidae). Sociobiology, 16, 285-287. 1990.

STRAND, M. R. Oviposition Behavior and Progeny Allocation of the Polyembryonic Wasp Copidosoma floridanum (Hymenoptera: Encyrtidae). Journal of lnsect Behavior, v. 2, n. 3. 1989.
TAYLOR, R. W.; JAISSON, P.; NAUMANN, I. D.; SHATTUCK, S. O. Notes on the biology of Australian bulldog ants (Myrmecia) and their chalcidoid parasites of the genus Austeucharis Boucek (Hymenoptera: Formicidae: Myrmeciinae: Eucharitidae: Eucharitinae). Sociobiology, v. 23, n. 2, p. 109-114. 1993.

THOMPSON, F. C. The Diptera site. The biosystematics database of world Diptera. Nomenclator status statistics. Version 1 0.5. <http://www.sel.barc. usda.gov/diptera/ names/ Statuslbdwdstat.htm >. Acesso: jan/2015. 2008.

TINAUT, A.; RUANO F. Parasitismo Social. Boletín de la Sociedad Entomológica Aragonesa. 6: 727-740. 1999.

TORRÉNS, J. A Review of the Biology of Eucharitidae (Hymenoptera: Chalcidoidea) from Argentina.

Psyche: A Journal of Entomology, v. 2013. doi:10.1155/2013/926572. 2013.

TORRENS, J.; HERATY, J. M. Description of the species of Dicoelothorax Ashmead (Chalcidoidea, Eucharitidae) and biology of D. platycerus Ashmead. ZooKeys. v. 165, p. 33-46. 2012.

TRJAPITZIN, V.A. A review of encyrtid wasps (Hymenoptera, Chalcidoidea, Encyrtidae) of Macaronesia, Entomological Review, v. 88, n. 2, p. 218-232, 2008.

UCD - UNIVERSAL CHALCIDOIDEA DATABASE. Perilampidae - Biology. 2004 http://www.nhm.ac.uk/ research-curation/research/projects/chalcidoids. Acessado em: 25 de março de 2014.

VANDER MEER, R. K.; JOUVENAZ, D. P.; WOJCIK, D. P. Chemical mimicry in a parasitoid (Hymenoptera: Eucharitidae) of fire ants (Hymenoptera: Formicidae). Journal of Chemical Ecology. 15, 2247-2261. 1989

WASMANN, E. Kritisches Verzeichniss der myrmecophilin und termitophilen Arthropoden. Felix Dames, Berlin xi . 1894. p. 231.

WHEELER, D. E. Ectatomma tuberculatum: Foraging Biology and Association with Crematogaster (Hymenoptera: Formicidae). Annals of the Entomological Society of America, v. 79, . 2, p. 300 303(4). 1986.

WHEELER, W. M. The polymorphism of ants, with an account of some singular abnormalities due to parasitism. Bulletin of the American Museum of Natural History, vol. 23, pp. 1-93. 1907.

WHEELER, W. M. Ants: their Structure, Development and Behavior (Vol. 9). Columbia University Press. 1910. p. 264. 
WILSON, E. O.; HOLLDOBLER, B. The rise of the ants: A phylogenetic and ecological explanation. Proceedings of the National Academy of Sciences of the United States of America. 102(21): 7411 - 7414. 2005.

WILSON, E. O. The Insect Societies. Harvard Univ Press, Cambridge, 1971.p. 548.

WOOLLEY, J. B. Chapter 5. Aphelinidae. In: GIBSON, G.A.P., J.T. HUBER, AND J.B. WOOLLEY. Annotated Keys to the Genera of Nearctic Chalcidoidea (Hymenoptera). National Research Council of Canada Research Press, Ottawa, Canada. 1997. p. 134-150.
ZAPPALÀ, L.; HOY, M. A.; CAVE, R. D. Interactions between the red imported fire ant, the citrus leafminer, and its parasitoid Ageniaspis citricola (Hymenoptera: Encyrtidae): Laboratory and field evaluations, Biocontrol Science and Technology, 17:4, 353-363, DOI: 10.1080/09583150701213612.2007. 